ARTICLE

\title{
TAL effector driven induction of a SWEET gene confers susceptibility to bacterial blight of cotton
}

Kevin L. Cox ${ }^{1,2, \star}$, Fanhong Meng ${ }^{1,2, \star}$, Katherine E. Wilkins ${ }^{3}$, Fangjun Li ${ }^{1,2}$, Ping Wang ${ }^{1,2}$, Nicholas J. Booher ${ }^{3}$, Sara C.D. Carpenter ${ }^{3}$, Li-Qing Chen ${ }^{4}$, Hui Zheng ${ }^{3}$, Xiquan Gao ${ }^{2,5,6}$, Yi Zheng $^{7}$, Zhangjun Fei ${ }^{7}$, John Z. Yu ${ }^{8}$, Thomas Isakeit ${ }^{1}$, Terry Wheeler ${ }^{1,9}$, Wolf B. Frommer ${ }^{10, \dagger}$, Ping He $\mathrm{H}^{2,5}$, Adam J. Bogdanove ${ }^{3}$ \& Libo Shan ${ }^{1,2}$

Transcription activator-like ( $T A L)$ effectors from Xanthomonas citri subsp. malvacearum $(X \mathrm{~cm})$ are essential for bacterial blight of cotton (BBC). Here, by combining transcriptome profiling with TAL effector-binding element (EBE) prediction, we show that GhSWEET10, encoding a functional sucrose transporter, is induced by Avrb6, a TAL effector determining $\mathrm{Xcm}$ pathogenicity. Activation of GhSWEET10 by designer TAL effectors (dTALEs) restores virulence of $X \mathrm{~cm}$ avrb6 deletion strains, whereas silencing of GhSWEET10 compromises cotton susceptibility to infections. A BBC-resistant line carrying an unknown recessive $b 6$ gene bears the same EBE as the susceptible line, but Avrb6-mediated induction of GhSWEET10 is reduced, suggesting a unique mechanism underlying b6-mediated resistance. We show via an extensive survey of GhSWEET transcriptional responsiveness to different $X_{c m}$ field isolates that additional GhSWEETs may also be involved in BBC. These findings advance our understanding of the disease and resistance in cotton and may facilitate the development cotton with improved resistance to BBC.

\footnotetext{
${ }^{1}$ Department of Plant Pathology and Microbiology, Texas A\&M University, College Station, Texas 77843, USA. ${ }^{2}$ Institute for Plant Genomics and Biotechnology, Texas A\&M University, College Station, Texas 77843, USA. ${ }^{3}$ Plant Pathology and Plant-Microbe Biology Section, School of Integrative Plant Science, Cornell University, Ithaca, New York 14853, USA. ${ }^{4}$ Department of Plant Biology, School of Integrative Biology, University of Illinois at UrbanaChampaign, Champaign, Illinois 61801, USA. ${ }^{5}$ Department of Biochemistry and Biophysics, Texas A\&M University, College Station, Texas 77843, USA. ${ }^{6}$ State Key Laboratory of Crop Genetics and Germplasm Enhancement, College of Agriculture, Nanjing Agricultural University, Nanjing 210095, China. ${ }^{7}$ Boyce Thompson Institute, Cornell University, Ithaca, New York 14853, USA. ${ }^{8}$ USDA-ARS, Southern Plains Agricultural Research Center, College Station, Texas 77845, USA. ${ }^{9}$ Texas Agricultural Experiment Station, Lubbock, Texas 79403, USA. ${ }^{10}$ Carnegie Science, Department of Plant Biology, 260 Panama Street, Stanford, California 94305, USA. † Present address: Institute of Molecular Physiology, Heinrich Heine University Düsseldorf and Max Planck Institute for Plant Breeding Research, 50829 Köln, Germany. * These authors contributed equally to this work. Correspondence and requests for materials should be addressed to A.J.B. (email: ajb7@cornell.edu) or to L.S. (email: Ishan@tamu.edu).
} 
C otton (Gossypium spp.) is an economically important crop and provides a significant source of fibre, feed, foodstuff, oil and biofuel products worldwide. The cotton genus is composed of at least 45 diploid and 5 tetraploid species ${ }^{1}$. The tetraploid (AD genome) species, including G. hirsutum that produces $95 \%$ of the world's cotton fibre, is likely derived from a hybridization between an A-genome-like ancestral species resembling $G$. arboreum and a D-genome-like ancestral species resembling G. raimondii ${ }^{2}$. The availability of the draft genome sequences for $G$. raimondii, G. arboreum, G. hirsutum and G. barbadense not only provides genetic resources to study the complex genome evolution and polyploidization process, but also lays the foundation for functional genomic approaches to dissect cotton gene functions with a goal to improve its agricultural performance in the face of biotic and abiotic stresses ${ }^{3-8}$.

Bacterial blight of cotton (BBC), caused by Xanthomonas citri subsp. malvacearum $(\mathrm{Xcm})$, is among the destructive diseases of cotton ${ }^{9}$. Following epidemics in the 1970s, the disease has occurred sporadically in the United States, but in the past several years re-emerged as a significant yield constraint. There are pressing needs to address the underlying mechanisms of susceptibility and resistance to $\mathrm{BBC}$ in cotton. $\mathrm{Xcm}$ injects effector proteins into plant cells via the type III secretion system to promote pathogenicity in plants. In the presence of corresponding resistance (R) proteins, some of these effectors trigger resistance and function as avirulence proteins. Interestingly, all known pathogenicity and avirulence factors (encoded by so called 'pth' and 'avr' genes) of $\mathrm{Xcm}$ are transcription activator-like (TAL) effectors ${ }^{10}$. TAL effectors functionally resemble eukaryotic transcription factors and upregulate host genes by directly binding to their promoters ${ }^{11,12}$. TAL effectors are highly conserved among different Xanthomonas spp., with an N-terminal type III translocation signal, a central repeat region (CRR) and C-terminal nuclear localization signals followed by an acidic activation domain. The proteins differ mainly in the CRR, which consists of 1.5-33.5 copies of near-perfect repeats of 33-34 amino acids. These repeats are conserved with the exception of the 12th and 13th residues of each copy, defined as the repeat variable di-residue (RVD) ${ }^{13,14}$. Each RVD targets a specific nucleotide of the promoters of host genes, creating a code, such that the sequence of RVDs defines the effector-binding element (EBE); each EBE starts, however, with a nearly invariant thymine, which is specified in a yet unclear manner by structures immediately $\mathrm{N}$ terminus of the $\mathrm{CRR}^{13-16}$.

Among the identified TAL effector targets include a group of pepper genes upregulated by AvrBs3 from $X$. campestris pv. vesicatoria $(X c v)^{17,18}$. These include the $R$ gene Bs3 and UPA20, which encodes a basic helix-loop-helix transcription factor regulating plant cell hypertrophy ${ }^{19}$. Os8N3/Xa13/OsSWEET11, essential for reproductive development, is a rice susceptibility $(S)$ gene targeted by TAL effector PthXo1 from X. oryzae pv. oryzae $(X o o)^{20}$. The xal3 allele, which is unable to be induced by PthXo1, acts as a recessive $r$ gene against Xoo infection ${ }^{21,22}$. Notably, four Os8N3/Xa13/OsSWEET11 homologues, rice Os11N3/OsSWEET14 and Xa25/OsSWEET13, pepper UPA16 and cassava MeSWEET10a are also targeted by TAL effectors from Xoo, Xcv and X. axonopodis pv. manihotis (Xam), respectively ${ }^{23-25}$. It was hypothesized that Xanthomonas spp. induces the expression of SWEET genes during infection to transport sucrose to the apoplast, thereby providing the bacteria with a carbon source $20,23,25-30$. TAL effectors from $X$. citri sp. citri $(X c c)$, the causal agent for citrus bacterial canker, induce the expression of CSSWEET1 in citrus, but the CsSWEET1 does not contribute demonstrably to susceptibility; another TAL effector target, however, CsLOB1, does function as an $S$ gene, promoting the characteristic pustule formation and bacterial multiplication ${ }^{31}$. Other characterized TAL effector targets include the rice bacterial blight $R$ genes $X a 27$ (ref. 32), Xa23 (ref. 33) and $X a 10$ (ref. 34), the bacterial leaf streak of rice $S$ gene OsSULTR3;6 (ref. 35) and the pepper bacterial spot $R$ gene $B s 4 C$ (ref. 36).

Previous work identified at least 10 TAL effectors from the cotton pathogen $\mathrm{Xcm} \mathrm{H} 1005$ and showed that a mutant with a deletion of seven tal genes was no longer able to cause observable water-soaking symptoms on Acala44 (Ac44), a BBC susceptible line of cotton. Avrb6 from $\mathrm{XcmH} 1005$ was among the first examples of an individual TAL effector important for $X c m$ virulence. An avrb6 mutant of $\mathrm{XcmH1005,XcmH1407}$

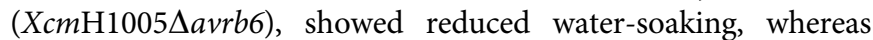
when expressed in a relatively weak virulent strain $X \mathrm{cmN1003,}$ Avrb6 enhances water-soaking ${ }^{37}$. Although it does not affect in planta bacterial multiplication, Avrb6 plays a major role in release of bacteria from the leaf interior to the leaf surface during infections ${ }^{37}$.

To identify the TAL effectors responsible for virulence and their targets, we sequenced the whole genomes of $\mathrm{Xcm}$ strains $X c m \mathrm{H} 1005$ and $X \mathrm{cmN} 1003$ and assembled the full repertoire of TAL effectors. Further, we deployed genome-wide gene expression profiling in cotton coupled with TAL effector DNA-binding code-assisted EBE prediction and identified the clade III sucrose transporter gene GhSWEET10 as a target of Avrb6. We determined that GhSWEET10 is a major $S$ gene for BBC by using dTALEs to induce it independently of any other possible Avrb6 targets and by silencing it with Agrobacteriummediated virus-induced gene silencing (VIGS). Significantly, other members of the clade III GhSWEET genes are strongly induced by different $X \mathrm{~cm}$ field strains responsible for recent re-emergence of the disease in the southern United States. Our data indicate that cotton GhSWEET10 and likely other SWEET sugar transporters in the same clade play a major role in BBC and suggest that newly evolved or horizontally transferred TAL effectors targeting these genes attribute to the re-emergence of $\mathrm{BBC}$ in the field. In addition, a BBC-resistant cotton line carrying $b 6$, a genetically complex resistance gene that has not yet been molecularly identified, showed markedly reduced Avrb6-mediated induction of GhSWEET10, ostensibly due to promoter polymorphisms outside the EBE. This suggests a novel mechanism for resistance that may represent a genetic determinant of $b 6$.

\section{Results}

Genome and TAL effector sequences of $\mathrm{XcmH1005}$ and N1003. The strains $X c m H 1005$, a rifamycin-resistant derivative of $X c m H$, and $X c m \mathrm{~N} 1003$, a spectinomycin- and rifamycin-resistant derivative of $X \mathrm{~cm} \mathrm{~N}$, are two $X \mathrm{~cm}$ strains that cause water-soaking on Ac44 cotton ${ }^{38}$. To set the stage for identifying the role that TAL effectors in these strains play in BBC, we generated complete genome sequences using single molecule real-time sequencing (Pacific Biosciences; hereafter 'PacBio sequencing'). The H1005 genome consists of a circular chromosome of 5,212,564 bp and a plasmid, $\mathrm{pXcmH}^{39}$ of $88,283 \mathrm{bp}$. The $\mathrm{N} 1003$ genome consists of a circular chromosome of 5,218,607 bp and a plasmid, herein designated as $\mathrm{pXcmN}$, of $59,644 \mathrm{bp}$. The general features of each genome are presented in Table 1 . The overall architectures of the two chromosomes are highly similar, with a single, large inversion (Fig. 1a). The plasmids are dissimilar, with only a few, relatively small regions of homology (Fig. 1a). The tal genes of the two strains are largely distinct (Fig. 1b and Supplementary Table 2). H1005 encodes 12 TAL effectors with six in the chromosome and six in $\mathrm{pXcmH}$. Ten of these are Avr proteins named previously based on reactions of differential cotton lines to transformants of 
Table 1 | Genome characteristics of $\mathrm{XcmH1005}$ and $\mathrm{XcmN1003.}$

\begin{tabular}{lll} 
Characteristics & $\mathbf{X c m H 1 0 0 5}$ & $\mathbf{X c m N 1 0 0 3}$ \\
\hline Length (bp) & $5,212,564 \mathrm{bp}$ & $5,218,607 \mathrm{bp}$ \\
No. of protein-coding genes & 4,227 & 4,218 \\
GC content & $64.62 \%$ & $64.54 \%$ \\
No. of tRNA genes & 54 & 54 \\
No. of rRNA operons & 2 & 2 \\
Components & Circular chromosome (5.2 Mb); plasmid (88,283 bp) & Circular chromosome (5.2 Mb); plasmid (59,644bp) \\
No. of TAL effectors (Chromosome::Plasmid) & $6:: 6$ & $5:: 4$ \\
GenBank accession no. & CP013004 & CP013006 \\
\end{tabular}
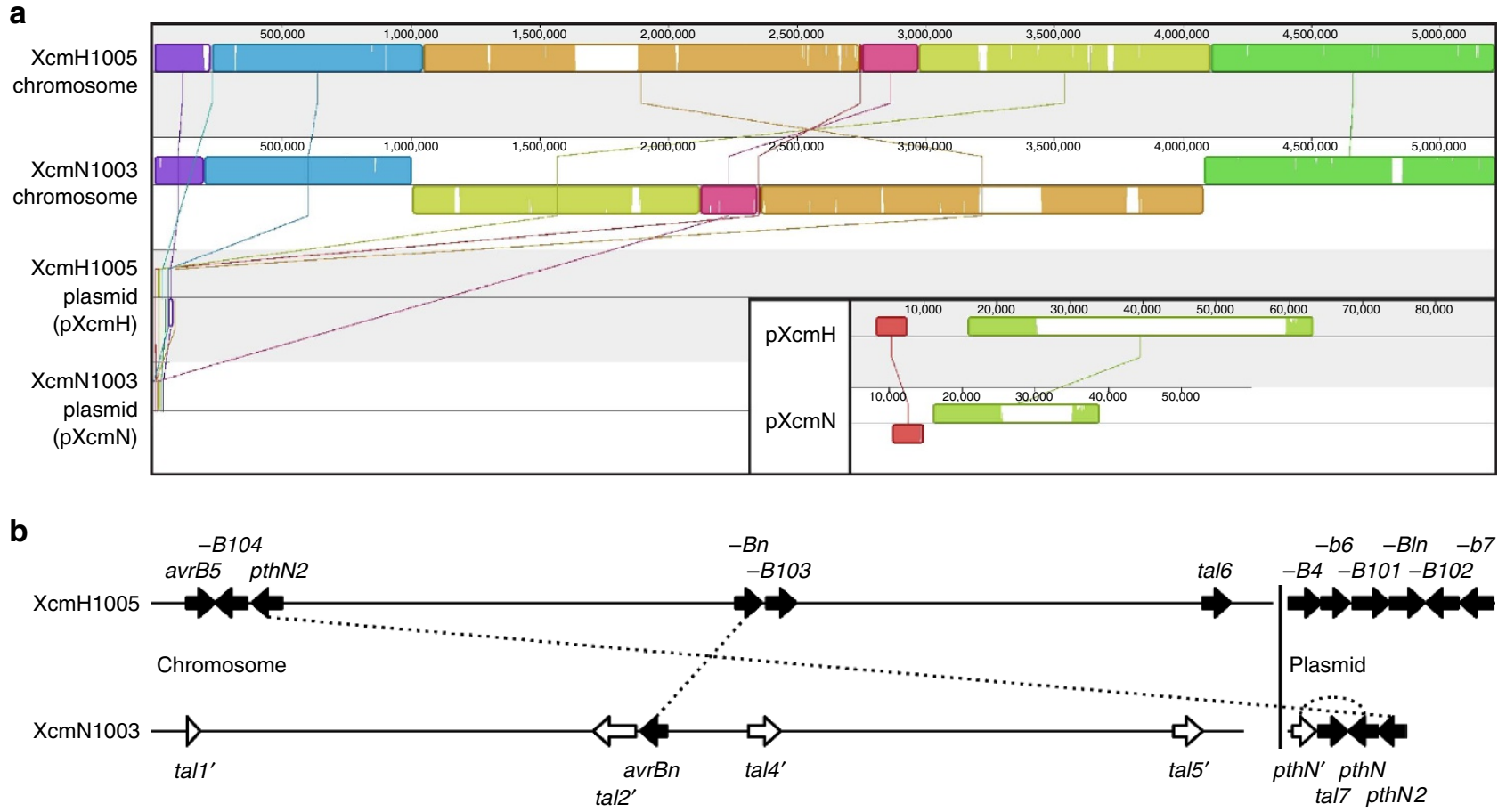

Figure 1 | Comparison of whole genomes and tal genes of $\mathbf{X c m H 1 0 0 5}$ and $\mathbf{X c m N 1 0 0 3 . ~ ( a ) ~ A l i g n m e n t ~ o f ~ t h e ~} X_{\mathrm{cm}} \mathrm{H} 1005$ and $X_{\mathrm{cmN}} \mathrm{N} 1003$ genomes, generated using progressiveMAUVE with default parameters ${ }^{68}$. Each genome comprises a single circular chromosome and a single circular plasmid, shown linearized. Coloured, rounded rectangles represent locally collinear blocks (LCB), regions of homology without rearrangement across the aligned sequences, connected by matching coloured diagonal lines. The orientations of the LCB, forward or reverse, are indicated by their position above or below the line, respectively. The height of a column within a block reflects the average similarity relative to the other aligned sequence(s) there (see http://darlinglab.org/ mauve/user-guide/viewer.html for details). Inset (bottom right) shows the alignment of the plasmids only, at a larger scale. Horizontal axes show sequence coordinates (bp). MAUVE backbone files giving the exact coordinates of all LCB are provided as Supplementary Data 1 (all molecules aligned) and Supplementary Table 1 (plasmids only). (b) The tal genes of $X_{c m H 1005}$ and $X_{c m} \mathrm{~N} 1003$. The genes are represented as block arrows at their relative positions in the chromosome or plasmid (horizontal lines). The arrows are magnified relative to the rest of the genome, but intergenic regions and arrow sizes relative to each other are to scale. Dashed lines connecting two arrows indicate identical encoded RVD sequences. Gene names follow the scheme of ref. 41, except for the indicated avr and pth genes, named previously 10,38-40. For label clarity, hypens replace 'avr'. An apostrophe following the gene name (white arrows) indicates that the coding sequence is terminated early due to a frameshift mutation or other coding sequence disruption. Details, including RVD sequences and AnnoTALE designations ${ }^{53}$, are given in Supplementary Table 2.

an otherwise-compatible $X \mathrm{~cm}$ strain carrying corresponding cosmid subclones: AvrB4, AvrB5, Avrb6, Avrb7, AvrB101, AvrB102, AvrB103, AvrB104, AvrBIn and AvrBn ${ }^{38-40}$. Another is PthN2, originally characterized in $X c m N 1003$ (ref. 10). The twelfth is uncharacterized, and we designated it as Tal6 $\mathrm{XcmH1005}_{\mathrm{c}}$, following a previously described naming scheme ${ }^{41}$. XcmN1003 harbours nine tal genes, five on the chromosome and four on $\mathrm{pXcmN}$. Four on the chromosome and one on the plasmid however are disrupted by a frameshift mutation or a large insertion in the repeat region or the $3^{\prime}$ end of the gene. One of the disrupted genes, tall', on the chromosome, carries only a last repeat and is otherwise complete until an integrase insertion further downstream. Another of the disrupted genes, $p t h N^{\prime}$ on $\mathrm{pXcmN}$, is otherwise identical to $p t h N$, an $X c m N 1003$ tal gene shown previously to contribute to water-soaking without triggering resistance on commercial US cotton varieties ${ }^{10}$. The intact $p t h N$ gene is also located on $\mathrm{pXcmN}$, along with $p t h N 2$, which also contributes to water-soaking, but less strongly ${ }^{10}$ Another intact tal gene, $t a l 7_{X c m N 1003}$, also resides on the plasmid. The fourth intact gene is avrBn located on the chromosome. Apart from $p t h N$, pthN, pthN2 and $a v r B n$, no other $X c m N 1003$ tal genes show obvious similarity in their RVD sequences to any other tal genes in either strain. The divergence in tal gene content between the two strains, both with respect to RVD composition 


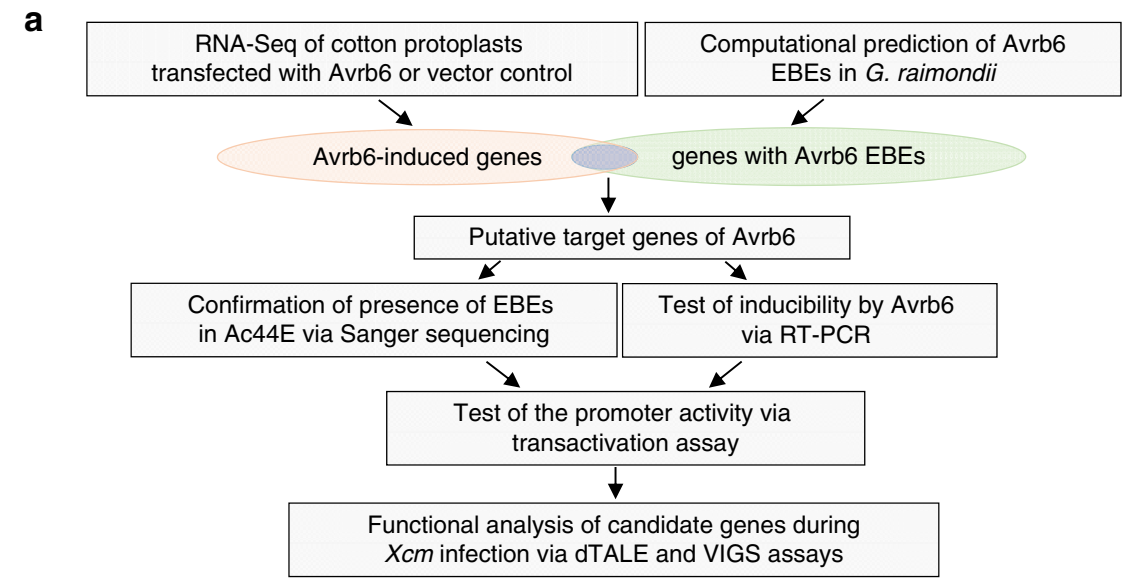

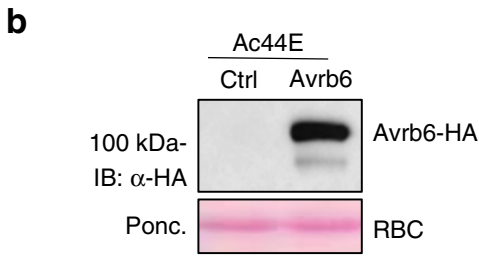

d

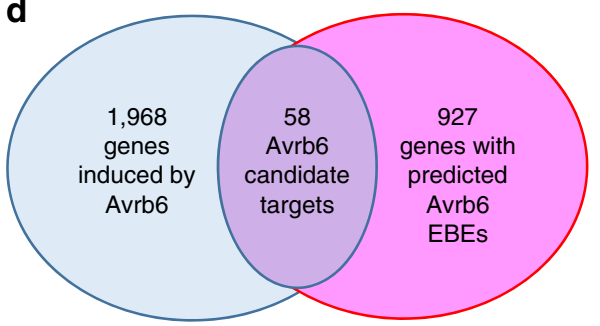

C

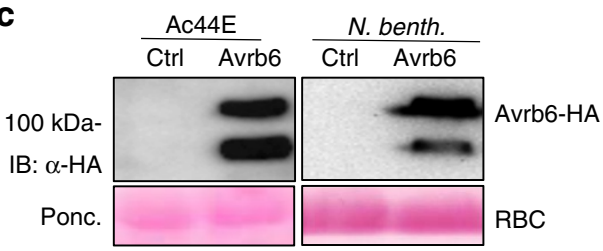

e

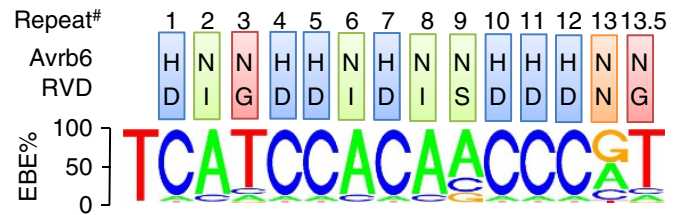

Figure 2 | Transcriptome profiling coupled with EBE prediction reveals candidate target genes of Avrb6. (a) Schematic diagram of experimental design to identify Avrb6 target genes in cotton. (b) Avrb6 protein expression in cotton protoplasts. Cotton protoplasts isolated from Ac44E were transfected with avrb6-HA or an empty vector as a control (Ctrl). Samples were collected $12 \mathrm{~h}$ after transfection and subjected to immunoblotting with $\alpha$-HA antibody (top panel). Ponceau S. staining (Ponc.) of total protein served as the protein loading control; RuBisCo (RBC) is shown (bottom panel). (c) Avrb6 protein expression in cotton and N. benthamiana. Cotyledons from 2-week-old Ac44E cotton and leaves of 4-week-old N. benthamiana were infiltrated with Agrobacterium carrying 35S:::avrb6-HA or an empty vector control (Ctrl). Inoculated tissues were collected at 48 hpi and subjected to immunoblotting. $\mathrm{B}$ and $\mathrm{C}$ were repeated three times with similar results. (d) Venn diagram of Avrb6-induced genes and genes with Avrb6 EBEs in G. raimondii genome. (e) The predicted EBE of Avrb6 RVDs. Coloured boxes on the top panel display the RVD of each repeat of Avrb6. Bottom panel indicates the relative frequencies of RVD associations with the four nucleotides for each repeat.

and genomic location of orthologs, is striking, which is in line with the fact that they were isolated from geographically distant regions ${ }^{38}$.

Candidate targets of Avrb6. Avrb6 is a TAL effector in $\mathrm{Xcm} \mathrm{H} 1005$ that causes strong water-soaking in the cotton line Ac44 (ref. 37). In this study, we used the Ac44E-tetraploid genotype, which is a single plant selection from Brinkerhoffs original Ac44 parent ${ }^{42}$. Ac44E has similar morphology to Ac44 except it has more abundant flowers and is slightly more susceptible to BBC than Ac44 (ref. 42). To identify the cotton genes that are specifically activated by Avrb6, we used an integrative approach involving a whole-genome RNA-sequencing (RNA-Seq) analysis of protoplasts of Ac44E expressing Avrb6 and computational prediction of Avrb6 EBEs in cotton (Fig. 2a). Compared to the whole plant-pathogen infection assay, the homogenous protoplast mesophyll cell system expressing the individual bacterial effector circumvents the complication of multiple pathogen effectors and elicitors that could spontaneously activate or suppress a large number of host genes. We cloned the full-length coding sequence of Avrb6 from Xcm H1005 into a plant expression vector with an HA-epitope tag at the $\mathrm{C}$ terminus under the control of the $35 \mathrm{~S} \mathrm{CaMV}$ promoter. The sequence was confirmed via Sanger sequencing (Supplementary Fig. 1). An immunoblot analysis with an $\alpha$-HA antibody detected a major polypeptide slightly above the $100 \mathrm{kDa}$ marker, which matches the predicted molecular mass of $108 \mathrm{kDa}$ for Avrb6 (Fig. 2b). We further subcloned the 35S::avrb6-HA expression cassette into a binary vector and performed Agrobacteriummediated transient expression assays in Ac44E cotton and Nicotiana benthamiana. As shown in Fig. 2c, we observed a similar result as in cotton protoplasts with a major polypeptide being detected at $\sim 108 \mathrm{kDa}$. Next, we performed RNA-Seq analysis with cotton protoplasts $12 \mathrm{~h}$ after transfection with the avrb6 construct or a vector control. Approximately 39-50 million raw read pairs and 36-46 million cleaned read pairs were obtained for each sample, which corresponds to $\sim 450 \times$ coverage of 77,267 annotated protein-coding transcripts ${ }^{1}$. Using a twofold expression change and an adjusted $P$ value $<0.05$ as cutoffs, and using the $G$. raimondii genome sequence as a reference (the tetraploid genome sequence was not available at the time), we identified 2,026 genes that were induced by Avrb6 (Fig. 2d and 


\begin{tabular}{|c|c|c|c|c|}
\hline Gene ID & Gene name & Induction fold & EBE prob. & Annotation \\
\hline Gorai.007G067700.1 & Gh067700 & 29.2 & 0.97 & Unknown protein \\
\hline Gorai.010G056300.1 & GhKBS1 & 9.6 & 0.96 & Kinase family protein \\
\hline Gorai.009G327300.1 & GhHLH1 & 4.0 & 0.89 & Basic helix-loop-helix TF \\
\hline Gorai.007G152300.1 & GhPPR1 & 5.2 & 0.64 & Pentatricopeptide repeat-containing protein \\
\hline Gorai.008G222500.1 & GhCYP1 & 471.6 & 0.59 & Cytochrome P450 \\
\hline Gorai.001G131000.2 & GhZFP1 & 12.3 & 0.51 & Zinc finger family protein \\
\hline Gorai.009G045100.2 & GhGLY1 & 12.8 & 0.5 & Glycine dehydrogenase \\
\hline
\end{tabular}

Supplementary Data 2). To identify genes with potential EBEs for Avrb6, we used the TALE-NT 2.0 Target Finder tool to examine all gene promoter sequences in the $G$. raimondii genome, using the standard score ratio cutoff of 3.0 (Fig. 2e). The list of genes displaying one or more putative EBEs was then intersected with the list of genes upregulated by Avrb6 to produce a list of candidate of Avrb6 targets (Table 2 and Supplementary Data 2). These candidates were ranked by the probability that the predicted EBE is functional by using a machine-learning algorithm based on the output of TALE-NT 2.0 and the genomic context of the predicted EBEs ${ }^{35}$. Interestingly, among the top candidates, Gorai.008G209000.1, a homologue of the SWEET sucrose transporter genes that serve as $S$ genes in rice and cassava, was induced $\sim 6,000$-fold by Avrb6 and has an EBE with a probability of 1 (Table 2). Other top candidates include Gorai.007G067700.1, Gorai.010G056300.1, Gorai.009G327300.1 and Gorai.008G047400.1. We then used the gene IDs from G. raimondii to identify their homologues in G. hirsutum. The gene IDs of the homologues identified in G. hirsutum are Gh_D12G1898 for Gorai.008G209000.1, Gh_D11G0631 for Gorai.007G067700.1, Gh_D06G0459 for Gorai.010G056300.1, Gh_D05G2954 for Gorai.009G327300.1 and Gh_D12G0420 for Gorai.008G047400.1. These genes were named GhSWEET10D (see below for explanation), Gh067700, GhKBS1, GhHLH1 and $G h M D R 1$, respectively, based on their predicted gene annotation.

Direct induction of targets by Avrb6. To independently determine the induction of the candidate genes, we performed reverse transcription (RT)-PCR assays using Ac44E cotton protoplasts transfected with Avrb6 or with an empty vector as control. The top five candidates, GhSWEET10, Gh067700, GhKBS1, GhHLH1 and $G h M D R 1$, were strongly induced by Avrb6 compared to the vector control (Fig. 3a). We further confirmed induction in cotton leaves by inoculating $X \mathrm{~cm}$ strains with or without avrb6. HM2.2S is derived from H1005 with a deletion of at least seven tal genes including avrb6, while HM2.2S (avrb6) is HM2.2S carrying avrb6 in plasmid vector pUFR127 (ref. 10). $\mathrm{H} 1005 \Delta a v r b 6$ is an avrb6 mutant derivative of $\mathrm{Xcm} \mathrm{H} 1005$ (ref. 37). As previously reported, avrb6 in different $X \mathrm{~cm}$ strains induced strong water-soaking on Ac44E cotton ${ }^{37}$ (Fig. 3b). We observed that water-soaking was only induced by the $X \mathrm{~cm}$ strains carrying avrb6 (HM2.2S (avrb6), HM2.2S (placZ::avrb6)— a strain that contains the plasmid pUFR135 which expresses avrb6 driven by the lacZ promoter, $\mathrm{H} 1005$ (placZ::avrb6) and H1005). As shown in Fig. 3c, the induction of GhSWEET10, Gh067700, GhKBS1 and GhMDR1 by the strains carrying avrb6, HM2.2S (avrb6) and H1005, was stronger than that by strains lacking avrb6, HM2.2S and H1005 Davrb6, at 12- and 24-h post inoculation (hpi). We could not amplify the GhHLH1 gene in cotton likely due to low transcript level. To explore whether GhSWEET10, Gh067700, GhKBS1 and GhMDR1 are directly induced by Avrb6, we added the eukaryotic protein synthesis inhibitor cycloheximide (CHX; $50 \mu \mathrm{M}$ final concentration) to the $\mathrm{Xcm}$ suspensions and inoculated into Ac44E cotton leaves. Each gene was still induced by strains carrying avrb6, H1005 and HM2.2S (avrb6), in the presence of CHX at 24 hpi (Fig. 3d), suggesting that new protein biosynthesis is not required for induction of these genes and that they are direct targets of Avrb6. The effectiveness of $\mathrm{CHX}$ in suppressing protein synthesis in this context was confirmed by its suppression of Avrb6 protein expression in a separate Agrobacterium-mediated transient expression assay in Ac44E cotton (Supplementary Fig. 2). To examine potential polymorphisms in these gene promoters between the sequenced cotton and Ac44E cotton used in this study, we amplified each promoter from Ac44E for sequencing. This revealed that each of the promoters possesses an Avrb6 EBE except Gh067700, which has a $1 \mathrm{bp}$ deletion in the EBE in Ac44E (Supplementary Fig. 3). To confirm whether Avrb6 activates the genes by targeting the EBEs, we amplified $\sim 800 \mathrm{bp}$ upstream of the translational start site of each gene, cloned each of these fragments upstream of a luciferase reporter gene $(L U C)$ and performed a cotton protoplast-based transactivation assay (Fig. 3e). Compared to an empty vector control, co-transfection of Avrb6 strongly activated pGhSWEET10D::LUC with about 110-fold induction, and also activated $p G h K B S 1:: L U C$ and $p G h M D R 1:: L U C$ with about 15-20-fold induction (Fig. 3f). Significantly, these promoters were not activated by PthN, another $X \mathrm{~cm}$ TAL effector with an RVD sequence distinct from Avrb6, indicating specificity of activation by Avrb6 (Fig. 3f). Consistent with the observation that $G$ h067700 in cotton Ac44E carries a nucleotide deletion in Avrb6 EBE, pGh067700::LUC was not activated by Avrb6. In addition, $p$ GhHLH1::LUC was not significantly activated by Avrb6, though its promoter possesses a predicted Avrb6 EBE. Since the promoter of GhSWEET10D was strongly induced by Avrb6, we tested the role of the EBE by mutating two nucleotides to create a version of $p$ GhSWEET10D::LUC with a 'CA' to 'GG' substitution in the EBE ( $m E B E$; Fig. $3 g$ ). Avrb6 strongly activated pGhSWEET10D::LUC carrying the wild-type EBE, but not the $m E B E$ (Fig. 3h). Taken together, these data indicate that Avrb6 directly and specifically activates GhSWEET10D, and likely GhKBS1 and GhMDR1, and that the EBE is required for Avrb6-induced expression of GhSWEET10D.

GhSWEET10 activation using a dTALE induces water-soaking. We generated dTALEs to specifically activate GhSWEET10D, 
a

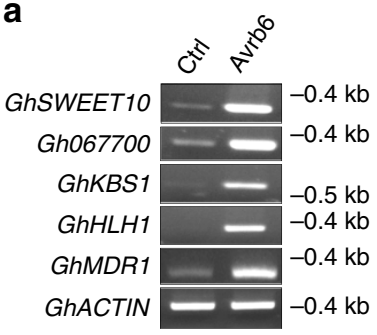

b

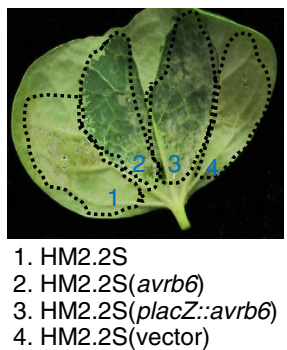

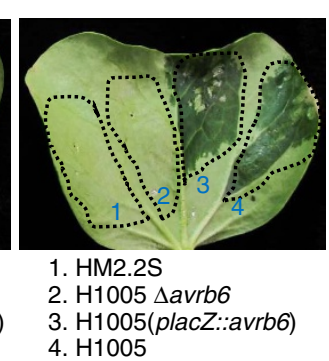

C

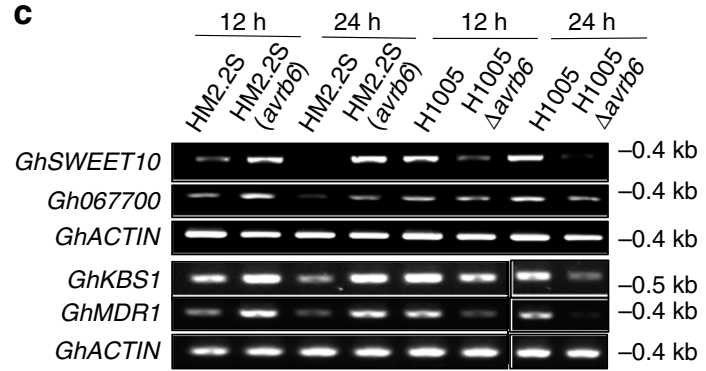

d

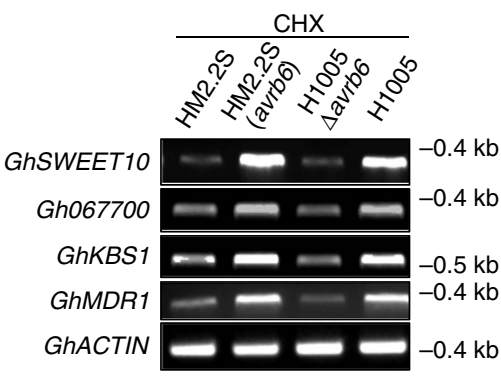

g

pGhSWEET10D

Wild-type EBE

TCCTCATCCCCAGCCCGTTCT

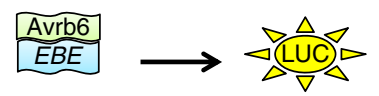

e

\begin{tabular}{|c|c|}
\hline $\begin{array}{l}\text { Effector plasmids } \\
\text { CaMV35S } a\end{array}$ & 26 HA NOS \\
\hline & \\
\hline $\begin{array}{l}\text { Reporter Plasmid } \\
\text { pGhSWEET10L }\end{array}$ & $-L U C-N O S$ \\
\hline pGh0677 & $-\angle U C-\sqrt{N O S}$ \\
\hline pGhKBS1 & LUC $-N O S$ \\
\hline pGhMDR1 & $-\angle U C-N O S$ \\
\hline pGhHLH1 & $\angle U C-N O$ \\
\hline
\end{tabular}

f
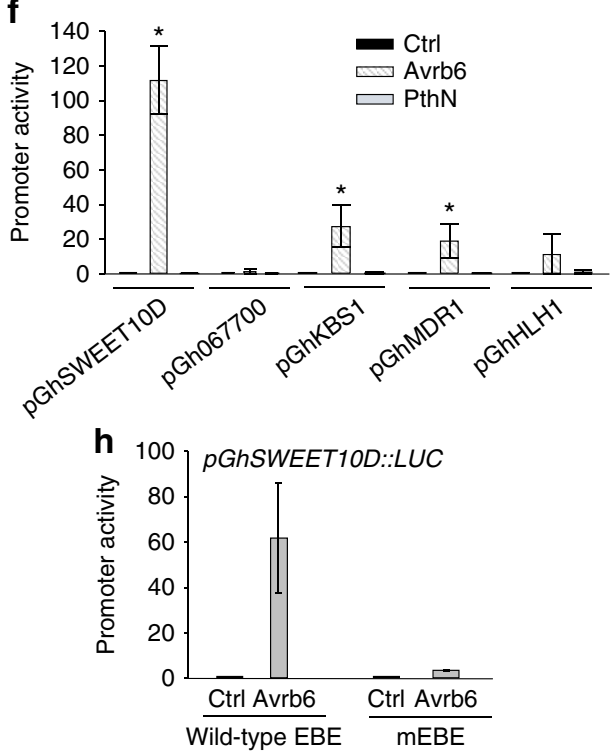

Figure 3 | Avrb6 directly induces cotton gene transcription in the absence of protein synthesis. (a) RT-PCR analysis of Avrb6 upregulated genes in cotton protoplasts. Cotton protoplasts of Ac44E were transfected with avrb6-HA or a vector control (Ctrl) and incubated for $12 \mathrm{~h}$ before RNA isolation. GhACTIN was used as an internal control. (b) Avrb6 contributes to water-soaking development in cotton. Cotyledons from 2-week-old Ac44E cotton were syringe-inoculated with different $X \mathrm{~cm}$ strains at $\mathrm{OD}_{600}=0.1$ and photographed 4 days after infiltration. Inoculation areas are indicated by dotted lines. (c) RT-PCR analysis of Avrb6 upregulated genes in cotton upon Xcm infection. Cotyledons from 2-week-old Ac44E cotton were syringe-inoculated with different $\mathrm{Xcm}$ strains. Tissues were collected at 12 and $24 \mathrm{hpi}$. (d) RT-PCR analysis of Avrb6 upregulated genes in the presence of CHX. Cotyledons from 2-week-old cotton were syringe-inoculated with different Xcm strains in $50 \mu \mathrm{M} \mathrm{CHX}$. Tissues were collected at $24 \mathrm{hpi}$ for RT-PCR. (e) Schematic diagram of the effector and reporter constructs. The reporter construct contains an expression cassette with a LUC reporter gene under the control of a candidate gene promoter. The effector construct contains either Avrb6 or PthN with an HA-epitope tag under the control of the CaMV 355 promoter. (f) Transcriptional activity of Avrb6 in cotton protoplasts. Protoplasts were co-transfected with a reporter construct and avrb6, pthN or a vector control (Ctrl), and were collected $12 \mathrm{~h}$ after transfection. UBQ10-GUS was included in the transfections as an internal control. The luciferase activity was normalized with GUS activity. The data are shown as mean \pm s.d. $(n=3)$ from three independent repeats. Asterisks indicate significant difference using two-tailed $t$-test $(P<0.05)$. ( $\mathbf{g})$ Schematic diagram of the transactivation assay of wild-type and mutated EBE (mEBE) in pGhSWEET10D in response to Avrb6. The nucleotide sequence containing the putative EBE is shown and the two nucleotides that were mutated are highlighted in blue. (h) Transcriptional activity of GhSWEET10D with wild type and mEBE in response to Avrb6. Cotton protoplasts were co-transfected with pGhSWEET10D::LUC carrying wild type or $\mathrm{mEBE}$ and Avrb6 or a vector control (Ctrl). The data are shown as mean \pm s.d. $(n=3)$ from three independent repeats. The above experiments were repeated three times with similar results.

GhKBS1 or GhMDR1 via EBEs distinct from the corresponding Avrb6 EBEs, introduced these into the Xcm HM2.2S strain, which lacks avrb6, and performed disease assays in cotton. The dTALEs were expressed from the low-copy, broad-host range vector, pKEB1, a derivative we constructed from pUFR047 (ref. 38) that carries a gateway cloning cassette (Fig. 4a). Significantly, HM2.2S transformants carrying the dTALE targeting GhSWEET10D (dTALE1), but not those targeting GhMDR1 (dTALE3) or GhKBS1 (dTALE4), or the vector control, induced water-soaking in Ac44E cotton, similarly to XcmH1005 (Fig. 4b). To determine if the dTALEs used indeed activate the transcripts of their respective target genes, we performed RT-PCR analysis. As shown in Fig. 4c, GhSWEET10D and GhMDR1 were induced by the cognate dTALEs. These data indicate that activation of
GhSWEET10D on $\mathrm{Xcm}$ infection contributes to water-soaking development, thus identifying GhSWEET10D as a relevant target of Avrb6 in cotton and an $S$ gene. We did not detect the induction of GhKBS1 by its dTALE, and cannot conclude whether GhKBS1 plays a role in BBC.

Avrb6 targets GhSWEET10 in both A and D genomes of cotton. The tetraploid G. hirsutum has two subgenomes, A and D. The above studies of GhSWEET10D were mainly based on the D genome of G. raimondii and the corresponding D subgenome of G. hirsutum. We examined if Avrb6 also targets SWEET10 in the A-genome of G. arboreum, which is closest to the progenitor species of the A subgenome of G. hirsutum. We identified a 
a

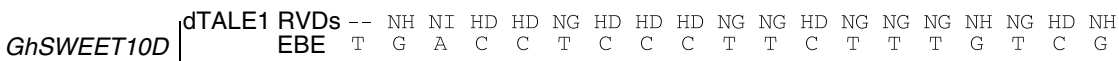

dTALE2 RVDs -- HD NG HD HD NG HD NH NH HD NG NI NG NI NI NI NG NI NG

$\begin{array}{llllllllllllllllllll}\text { EBE } & T & C & T & C & C & T & C & G & G & C & T & A & T & A & A & A & T & A & T\end{array}$

GhMDR1 dTALE3 RVDs -- HD NG HD NG NH NI HD NG NG HD NG NG NH NG HD NI NI NI HD NG

EBE
GhKBS1

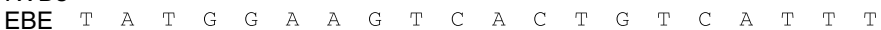

b
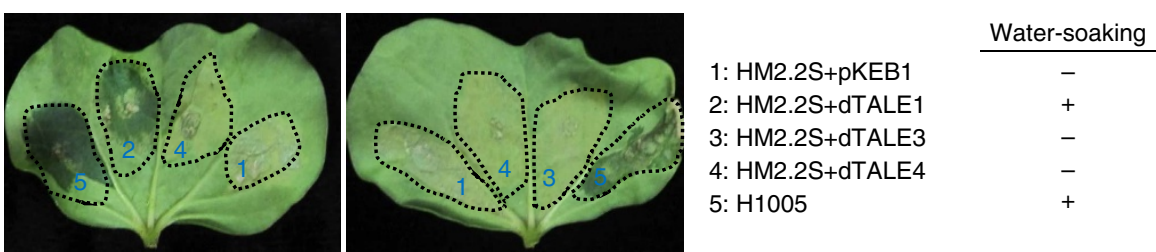

c
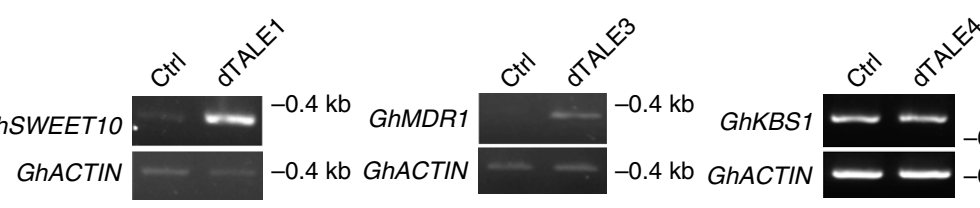

d

e

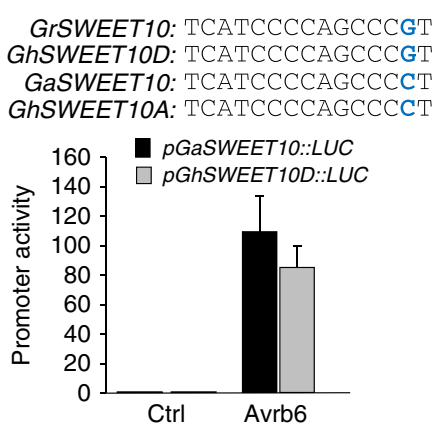

f GaSWEET10: TGACCTTCCTT----GTCG dTALE1: TGACCTTCCTTCTTTGTCG GaSWEET10: TCTCCTCGGCTATAAATAT dTALE2: TCTCCTCGGCTATAAATAT

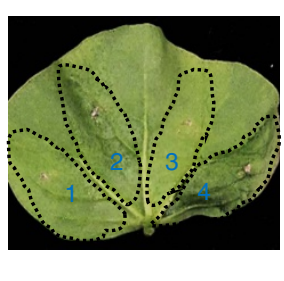

Figure 4 | The dTALE-activating GhSWEET10 induces water-soaking in cotton. (a) RVDs of dTALEs and the corresponding targeted EBE sequences in the G. hirsutum genome. (b) Xcm HM2.2S expressing a dTALE uniquely targeting GhSWEET10D induces water-soaking in cotton. Cotyledons from 2-week-old Ac44E plants were syringe-inoculated with different $X_{c m}$ strains at $O_{600}=0.1$ and photographed at 4 dpi. The table displays the presence $(+)$ or absence ( - ) of water-soaking. (c) RT-PCR analysis of GhSWEET10, GhKBS1 and GhMDR1 targeted by their respective dTALEs. Two-week-old cotyledons of Ac44E were syringe-inoculated with $X \mathrm{~cm}$ strains $\mathrm{HM} 2.2 \mathrm{~S}\left(\mathrm{OD}_{600}=0.1\right)$ containing different dTALEs. Inoculated tissues were collected at 24 hpi for RT-PCR analysis. (d) GaSWEET10 is induced by Avrb6 in G. arboreum protoplasts. Protoplasts isolated from G. arboreum were transfected with Avrb6 or an empty vector control (Ctrl) and samples were collected $12 \mathrm{~h}$ after transfection for RT-PCR analysis. (e) Transactivation assay of GaSWEET10 and GhSWEET10D promoters in response to Avrb6 in cotton protoplasts. Alignment of Avrb6 EBEs reveals a polymorphism between GhSWEET10D and GaSWEET10 promoters, which were highlighted in blue. Protoplasts isolated from commercial cotton variety FM706V were co-transfected with pGaSWEET10::LUC or pGhSWEET10D:::LUC and Avrb6 or a vector control (Ctrl). The data are shown as mean \pm s.d. $(n=3)$ from three independent repeats. (f) The dTALE matching the GaSWEET10 promoter causes water-soaking on G. arboreum. The EBE sequences of two dTALEs targeted to different regions of the GaSWEET10 promoter are shown. dTALE2 with an EBE sequence perfectly matching the GaSWEET10 promoter but not dTALE1 with a partially matching EBE sequence restores $X \mathrm{~cm}$-mediated water-soaking on cotton. Cotyledons from 2-week-old $\mathrm{G}$. arboreum were syringe-inoculated with different $X \mathrm{~cm}$ strains at $\mathrm{OD}_{600}=0.1$ and photographed at $4 \mathrm{dpi}$. The above experiments were repeated three times with similar results.

homologue of GhSWEET10D, named GaSWEET10, which bears $98 \%$ similarity to GhSWEET10D at the amino-acid level. We expressed Avrb6 in G. arboreum cotton protoplasts and determined whether GaSWEET10 was induced by Avrb6. RT-PCR analysis showed that GaSWEET10 was induced by Avrb6 (Fig. 4d). To determine whether Avrb6 could specifically activate the promoter of GaSWEET10, we cloned the promoter, $p G a S W E E T 10$, upstream of the luciferase gene for a transactivation assay. As shown in Fig. 4e, Avrb6 activated pGaSWEET10::LUC to a similar level as Avrb6 activation of pGhSWEET10D::LUC. Notably, an alignment of the Avrb6 EBE from $p G h S W E E T 10 D$ with the one from $p G a S W E E T 10$ revealed them to be identical except for a single nucleotide polymorphism near the $3^{\prime}$ end of the EBE (position 14, counting the initial thymine), where mismatches are known to be generally tolerated $^{43}$. Additionally, an alignment of protein-coding sequences revealed that GrSWEET10 from $G$. raimondii is identical to D subgenome GhSWEET10D, whereas GaSWEET10 from G. arboreum is identical to A subgenome GhSWEET10A (Supplementary Fig. 4). Similarly, the promoter sequence, including the EBE, of $p G h S W E E T 10 A$ from tetraploid Ac44E was more closely related to $p G a S W E E T 10$ from $G$. arboreum versus $p G r S W E E T 10$ from G. raimondii (Supplementary Fig. 4b). We used the dTALE approach to determine if GaSWEET10 is also a direct target of Avrb6 that causes water-soaking. As shown in Fig. 4f, the dTALE2 construct, but not the dTALE1 construct specific to $p G h S W E E T 10 D$ or an empty vector introduced in H1005 $\Delta$ avrb6-induced water-soaking in G. arboreum. Notably, 

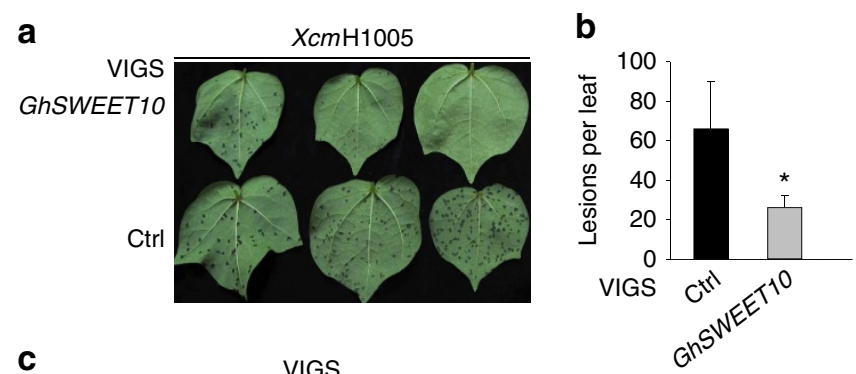

C

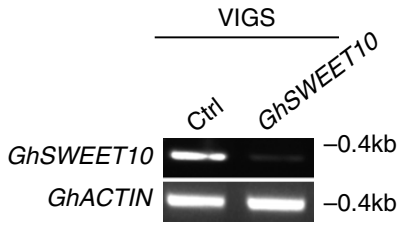

Figure 5 | Silencing of GhSWEET10 in cotton reduces water-soaking caused by $\mathbf{X c m}$ infection. (a) $\mathrm{Xcm}$-mediated water-soaking lesion development on cotton plants upon VIGS. Cotyledons from 2-week-old Ac44E plants were syringe-infiltrated with Agrobacterium carrying a VIGS construct to silence GhSWEET10 (VIGS-GhSWEET10) or a GFP vector control (VIGS-Ctrl). Three weeks later, plants were vacuum infiltrated with $X_{c m H 1005}$ at $\mathrm{OD}_{600}=0.01$. Images were taken 2 weeks after inoculation. (b) Quantitative analysis of $X \mathrm{~cm}$-mediated water-soaking lesions on cotton plants upon VIGS. Lesions were counted on the second true leaf of each VIGS plant (at least 10 plants were inoculated for each construct in each repeat). The data are shown as mean \pm s.e. $(n=10)$. The asterisk indicates a significant difference with a Student's $t$-test $(P<0.05)$ when compared with the control. (c) RT-PCR analysis of GhSWEET10 expression in cotton plants upon VIGS. VIGS assays were done similarly as in a and 3 weeks later, the second true leaf was collected for RT-PCR analysis before $X \mathrm{~cm}$ inoculation. GhACTIN was used as an internal control. The above experiments were repeated three times with similar results.

H1005 induced relatively weak water-soaking on G. arboreum compared to $G$. hirsutum, likely due to G. arboreum carrying some strain-specific resistance as noted previously ${ }^{44}$. Taken together, these results indicate that Avrb6 can directly target SWEET10 in both the A and D genomes to contribute to the development of BBC.

GhSWEET10 silencing reduces water-soaking caused by Xcm. Having determined that GhSWEET10 mediates Avrb6-induced water-soaking, we subsequently investigated whether GhSWEET10 is genetically required for water-soaking during $\mathrm{Xcm}$ infection in cotton. We employed the Agrobacterium-mediated VIGS system to silence GhSWEET10 and examined BBC development after subsequent inoculation of $X \mathrm{~cm}$. The VIGS construct was designed to silence both GhSWEET10A and GhSWEET10D because of sequence conservation. There was no apparent difference in plant growth between the VIGS-GhSWEET10 plants and plants inoculated with the GFP control (VIGS-Ctrl) up to 3 weeks after VIGS (Supplementary Fig. 5). Three weeks after Agrobacterium infiltration, the silenced plants were vacuum infiltrated with $X c m H 1005$. Compared to the control, the VIGS-GhSWEET10 plants consistently showed fewer water-soaked lesions after $X c m \mathrm{H} 1005$ infection (Fig. 5a,b). RT-PCR analysis confirmed reduced induction of GhSWEET10 in VIGS-GhSWEET10 plants compared to VIGS-Ctrl plants (Fig. 5c). Taken together, the data indicate that silencing of GhSWEET10 in cotton reduces the development of water-soaking following $X \mathrm{~cm}$ inoculation, providing genetic evidence that GhSWEET10 is functionally required for normal BBC symptom development upon infection by $\mathrm{Xcm}$ strains carrying avrb6.
GhSWEET10D encodes a functional sucrose transporter. Since GhSWEET10 belongs to the SWEET gene family, we examined whether GhSWEET10 functions as a sugar transporter. To measure activity, we transiently expressed the coding sequence of GhSWEET10D along with a Förster resonance energy transfer (FRET) sucrose sensor in human embryonic kidney T293 (HEK293T) cells ${ }^{45}$. Compared to the vector control, expression of GhSWEET10D enabled HEK293T cells to accumulate sucrose, which was detected as a negative ratio change, to a level comparable to the well-characterized Arabidopsis transporter AtSWEET11 (Fig. 6a). In addition, when we transiently expressed GhSWEET10D under the control of the 35S CaMV promoter in $N$. benthamiana, the sucrose concentration of the apoplastic fluids from the 35S::GhSWEET10D-inoculated leaves was higher than that from the vector control-inoculated leaves (Supplementary Fig. 6a), indicating that GhSWEET10D is able to transport sucrose in planta. Interestingly, GhSWEET10D did not restore the growth defect of yeast EBY4000, a hexose transport-deficient yeast strain, on the medium supplemented with $2 \%$ glucose or $2 \%$ fructose as the sole carbon source, indicating that GhSWEET10D does not mediate efficient fructose or glucose transport (Supplementary Fig. 6b). Taken together, the data suggested that GhSWEET10D encodes a functional sucrose transporter.

GhSWEET10D is a member of a large gene family in cotton. In bacterial blight of rice, several closely related SWEET genes can each serve as an $S$ gene ${ }^{28}$. To better understand the relationship within the SWEET family in cotton, especially which SWEETs are most closely related to GhSWEET10, the full-length protein sequence of GhSWEET10D was used as the query for a BLASTp analysis against the G. hirsutum NBI protein database (www.cottongen.org/tools/blast). The search identified 50 additional GhSWEETs, for a total of 51 SWEETs in G. hirsutum, with each possessing the conserved domains found in the AtSWEET superfamily in Arabidopsis (Supplementary Figs 7 and 8). Based on the well-characterized phylogenetic analysis of AtSWEETs, we generated a phylogenetic tree and classified the GhSWEET family into four clades with nine in clade I, 14 in clade II, 18 in clade III and 10 in clade IV. The individual members of GhSWEETs are named using the tree described by Eom et al. ${ }^{46}$, as a reference where GhSWEET1-3 are in clade I, GhSWEET4-8 are in clade II, GhSWEET9-15 are in clade III and GhSWEET16-18 are in clade IV. Considering the gene duplication and polyploidy in tetraploid cotton, lowercase and uppercase letters are used, respectively, to distinguish closely related members. If multiple members are co-orthologous to the closest AtSWEET, lowercase letters $(\mathrm{a}-\mathrm{c})$ are added after the numbers to differentiate genes in the same subclade. An uppercase ' $A$ ' is further added to indicate the gene is from the A subgenome, or an uppercase ' $D$ ' for the $\mathrm{D}$ subgenome. For example, there appear to be two pairs of GhSWEETs that are closely related to AtSWEET1 as shown in the phylogenetic tree. Therefore, we distinguish these by naming one pair as GhSWEET1a and another pair as GhSWEET1b. Each of these pairs is further named according to their subgenome locations as GhSWEET1aA and GhSWEET1aD, and GhSWEET1bA and GhSWEET1bD, respectively (Fig. $6 \mathrm{~b}$ and Supplementary Fig. 7). Like the SWEET genes in rice and cassava that serve as $S$ genes, GhSWEET10 is a member of the clade III family (Fig. 6c).

GhSWEET10 induction by Avrb6 is reduced in b6 cotton. Avrb6 causes severe water-soaking in the susceptible Ac44E cotton line. However, in the near-isogenic resistant line Acb6, 

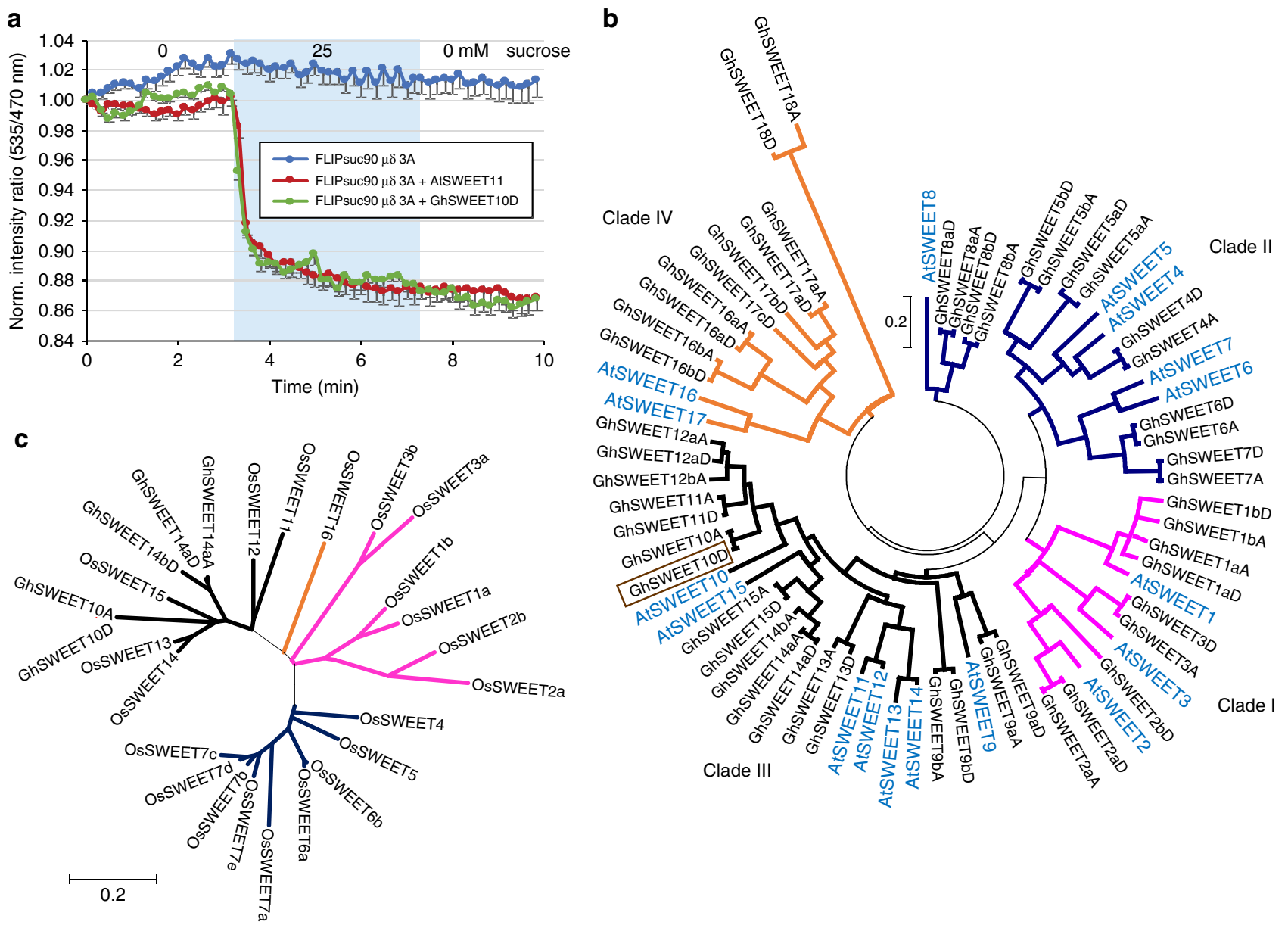

Figure 6 | GhSWEET10D encodes a functional clade III sucrose transporter. (a) Detection of GhSWEET10D transporter activity in HEK293T cells using the sucrose sensor FLIPsuc $90 \mu \delta 3 \mathrm{~A}$. Sucrose transporter activity was assayed by co-expressing GhSWEET10D with the cytosolic FRET sucrose sensor FLIPsuc $90 \mu \delta 3 \mathrm{~A}$ in HEK293T cells. Blue circles correspond to cells expressing the sensor alone; red circles and green circles correspond to cells co-expressing the sensor with AtSWEET11 or GhSWEET10D, respectively. The cyan block indicates duration of perfusion with 25 mM sucrose. Accumulation of sucrose is reported as a negative ratio change (mean - s.e.m.; $n>10$ ). Experiments were repeated four times with similar results. (b) Phylogenetic analysis of GhSWEET proteins from G. hirsutum. AtSWEET proteins from Arabidopsis thaliana were included to represent different clades of SWEET superfamily. The phylogenetic tree was made using the neighbour-joining method in MEGA version 6.06. The evolutionary distances were computed using the Poisson correction method and are in the units of the number of amino acid substitutions per site ${ }^{69}$. GhSWEET10D, which was used as the query, is highlighted with a brown box. (c) Phylogenetic analysis of GhSWEET10, GhSWEET14a, GhSWEET14b and the OsSWEET proteins from Oryza sativa. The phylogenetic tree was made as described in $\mathbf{b}$. The clades of the SWEET family were colour coded as follows: clade I= pink, clade II = blue, clade III = black and clade IV = orange.

which harbours the apparently genetically complex $\mathrm{BBC}$ recessive resistance locus b6, Avrb6 causes the resistance-associated hypersensitive response (HR) instead (ref. 38 and Supplementary Fig. 9a,b). We examined whether $b 6$-mediated resistance is in part due to the inability of Avrb6 to activate GhSWEET10 in Acb6. When we expressed Avrb6 in Ac44E and Acb6 protoplasts, respectively, the induction of GhSWEET10 by Avrb6 was significantly lower in Acb6 than that in Ac44E (Fig. 7a). We further compared GhSWEET10 induction in Ac44E and Acb6 cotton plants inoculated with different $\mathrm{Xcm}$ strains with or without avrb6. Both Ac44E and Acb6 plants showed increased induction of GhSWEET10 on inoculation with HM2.2S carrying avrb6 compared to the plants inoculated with HM2.2S (Fig. 7b). However, the induction was significantly reduced in Acb6 plants relative to Ac44E plants. A similar trend was observed when H1005 was used to inoculate both lines (Fig. 7b). To examine whether the reduced induction by Avrb6 in Acb6 could be due to polymorphisms at the GhSWEET1OD EBE, we sequenced the GhSWEET10D promoter region from Acb6. The EBE is perfectly conserved, but in Acb6, there is a SNP immediately adjacent to the $5^{\prime}$ of the EBE and a $2 \mathrm{bp}$ insertion surrounded by six additional SNPs $\sim 20$ bp downstream of the EBE (Supplementary Fig. 9c). We cloned the promoter of GhSWEET10D from Acb6 in front of the luciferase reporter for a transactivation assay in cotton protoplasts. Similar to what we observed for the endogenous GhSWEET10D gene, the induction of the

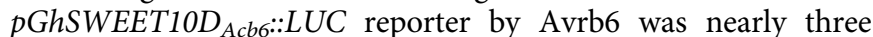
folds lower than that of the reporter carrying the promoter from Ac44E ( $p$ GhSWEET10D $\left.{ }_{A c 44 E}: L U C\right)$ (Fig. 7c). The promoter of GhSWEET10A is almost identical in Ac44E and Acb6 (Supplementary Fig. 4b). Thus, the polymorphisms outside the GhSWEET10D EBE may impair full induction by Avrb6. In addition, when we inoculated H1005 $\Delta a v r b 6$ carrying dTALE2, which activates pGhSWEET10D (Fig. 4a,f), into cotyledons of Acb6, the bacterium triggered water-soaking, but not HR in Acb6 (Supplementary Fig. 9d). The data suggest that GhSWEET10D may not mediate Avrb6-induced HR and Avrb6 targets another gene for HR in Acb6. 
a
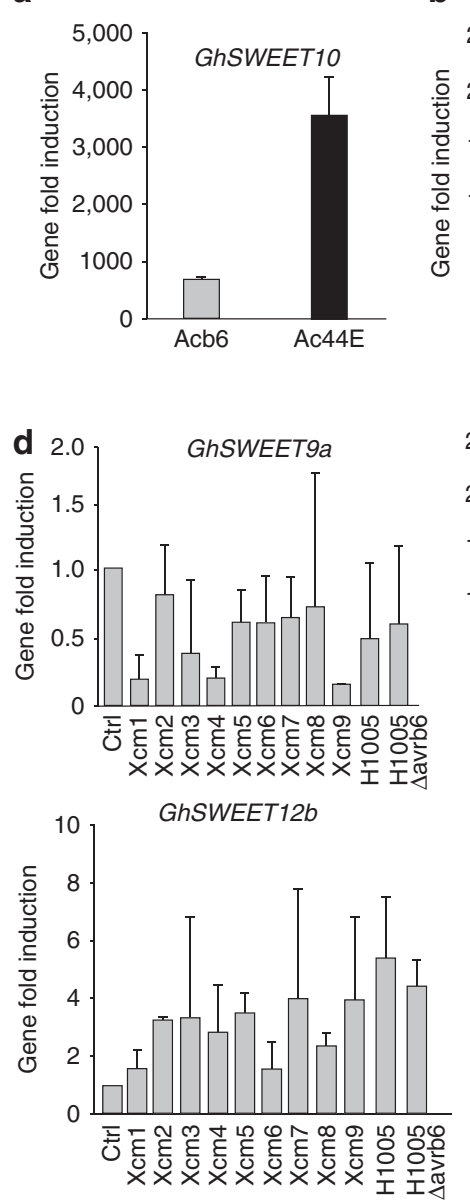

b

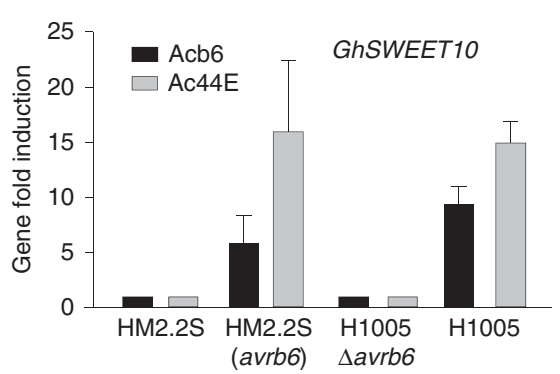

C

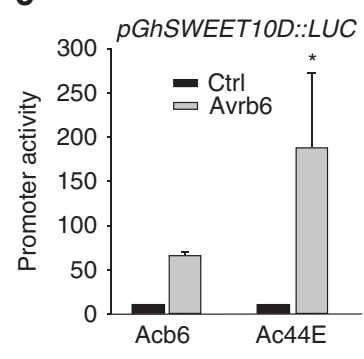

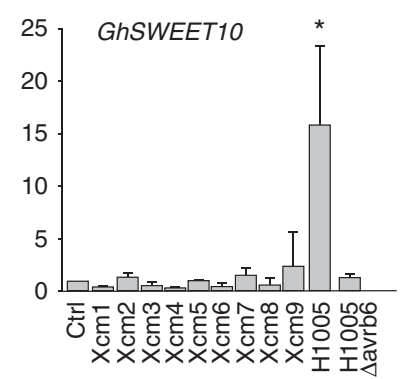

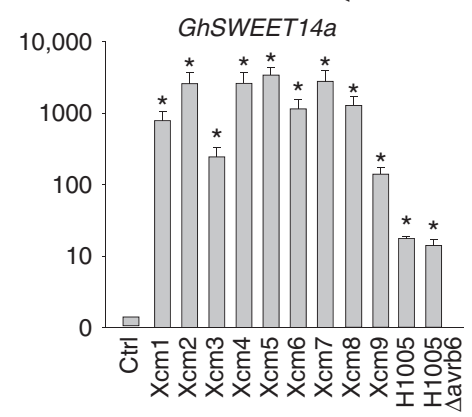

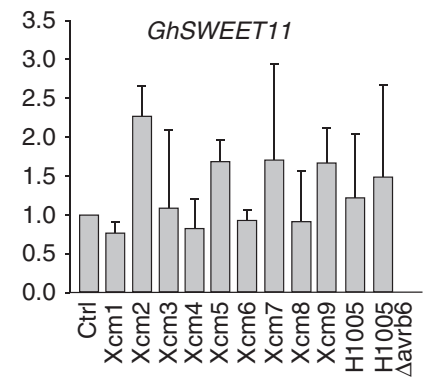
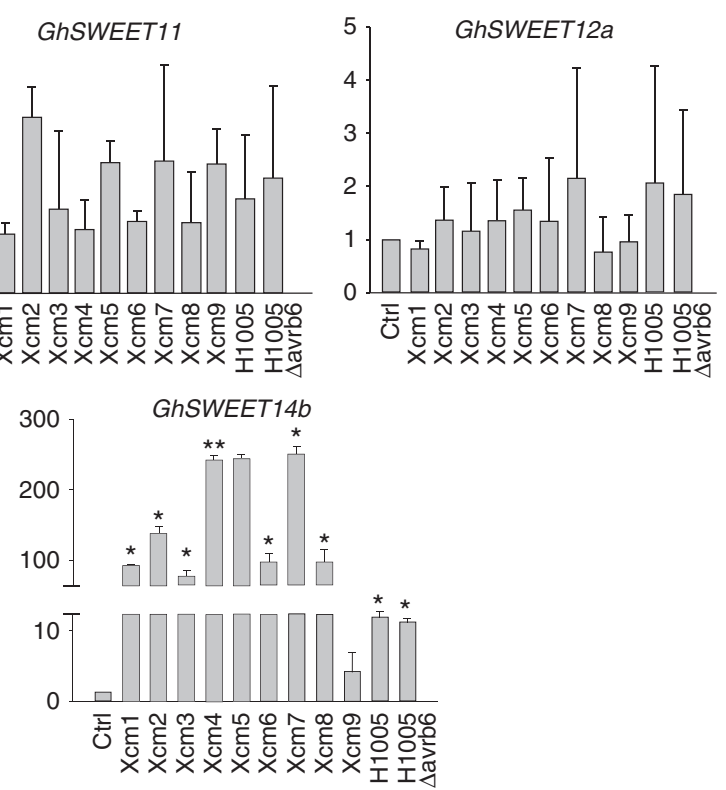

Figure 7 | Induction of GhSWEET genes in different cotton-Xcm interactions. (a) GhSWEET10 induction by Avrb6 in protoplasts of the resistant line Acb6 and the susceptible line Ac44E. Graph displays Avrb6-induced GhSWEET10 reads per kilobase of transcript per million mapped reads and fold change from RNA-Seq analysis with Ac44E (compatible) and Acb6 (incompatible) cotton lines. (b) qRT-PCR analysis of GhSWEET10 in Ac44E and Acb6 plants upon $X_{c m}$ infections. Two-week-old cotyledons were syringe-inoculated with different $X_{c m}$ strains at $\mathrm{OD}_{600}=0.1$ and tissues were collected at 24 hpi. GhUBQ1 was used as an internal control. The data are shown as mean \pm s.d. $(n=3)$ from three independent repeats. (c) Transactivation assay of $p G h S W E E T 10 D:: L U C$ from Acb6 and Ac44E in response to Avrb6 in cotton protoplasts. The promoter of GhSWEET10D was amplified from Acb6 and Ac44E, respectively, and fused with luciferase reporter. Protoplasts of cotton variety FM706V were co-transfected with the reporter construct and Avrb6 or an empty vector control $(\mathrm{Ctrl})$. The data are shown as mean \pm s.d. $(n=3)$ from three independent repeats. An asterisk indicates the significant difference using two-tailed $t$-test $(P<0.05)$ between two reporter constructs after Avrb6 induction. (d) Induction of clade III GhSWEET genes by different $X \mathrm{~cm}$ isolates. Two-week-old cotyledons were syringe-inoculated with different $X \mathrm{~cm}$ isolates $\left(X \mathrm{~cm} 1-X_{\mathrm{cm}} 9\right)$ collected from different locations in Texas, $\mathrm{USA}_{\mathrm{S}} \mathrm{OD}_{600}=0.1$. Tissues were collected at $24 \mathrm{hpi}$. GhUBQ1 was used as an internal control for qRT-PCR analysis. The data are shown as mean \pm s.d. $(n=3)$ from three independent repeats. An asterisk indicates significant difference using two-tailed $t$-test $(P<0.01)$ compared to the water control. The above experiments were repeated three times with comparable results.

Clade III GhSWEET responsiveness to different $\mathrm{Xcm}$ isolates. Clade III SWEET genes have been implicated in disease susceptibility in rice and cassava. We here identified a clade III SWEET gene in cotton, GhSWEET10, that encodes a sucrose transporter and that also plays a role in disease susceptibility. To examine how general the involvement of clade III GhSWEET genes in $\mathrm{BBC}$ is, we investigated the transcriptional changes of these genes in response to nine $\mathrm{Xcm}$ isolates collected from infected cotton fields from different locations in Texas. Notably, these isolates are suspected to be the causal agents of the recent rampant re-emergence of BBC in the United States. However, it has not been determined yet whether these isolates represent a new race. We found that the field isolates were unable to induce GhSWEET10. However, all of the field isolates highly induced GhSWEET14a (200-4,000-fold) (Fig. 7d). In addition, all of the isolates, except for Xcm9, also significantly induced GhSWEET14b (20-300-fold). As expected, H1005, but not H1005 induced GhSWEET10 by 15 -fold. Notably, GhSWEET14a and GhSWEET14b are clade III SWEET genes (Fig. 6c). Taken together, these data suggest that in addition to GhSWEET10, GhSWEET14a and GhSWEET14b are potential $S$ genes, and that activating clade III GhSWEET genes might be broadly important to the ability of different $X \mathrm{~cm}$ strains to cause BBC.

\section{Discussion}

BBC caused by $X \mathrm{~cm}$ is among devastating diseases that limit cotton yields. Despite several TAL effectors being cloned from $X c m$, no $R$ or $S$ genes have yet been molecularly identified, mainly because of the large and polyploidy nature of the cotton genome and limited molecular and genomic tools. The approach taken in this study successfully overcame these challenges to establish a role for SWEET-mediated susceptibility in BBC. GhSWEET10 was strongly induced by Avrb6 during Xcm 
infection and was identified as an $S$ gene for BBC. Characterization of BBC-resistant line Acb6 revealed sequence differences in the GhSWEET1OD promoter, but not in the EBE, that impair activation by Avrb6, possibly relating to resistance mediated by the $b 6$ gene. Finally, surveys of different $X c m$ field isolates showed that two additional clade III GhSWEETs were highly induced during infection. It appears that the evolution of novel TAL effectors that induce these additional clade III GhSWEET members may have contributed to the recent re-emergence of $\mathrm{BBC}$ in the United States.

The SWEET family members are key $S$ genes common to several diseases caused by different Xanthomonas species ${ }^{47}$. So far, only clade III SWEET genes are targeted by pathogens that use TAL effectors to facilitate disease ${ }^{46,48}$. Members of clade III SWEET genes are known to transport sucrose, which likely serves as a carbon source for the pathogen. Interestingly, it was recently shown that two clade III SWEET genes in Arabidopsis, AtSWEET13 and AtSWEET14, can also transport plant hormone gibberellin to modulate growth and development ${ }^{49}$. In rice, Xoo can target three clade III SWEET genes to cause susceptibility. Xoo TAL effector PthXo1 targets OsSWEET11 (ref. 20); PthXo2 targets OsSWEET13 (ref. 27); and AvrXa7 targets OsSWEET14 (ref. 23). In cassava, Xam can use TAL20 Xam668 to target MeSWEET10a, another clade III SWEET gene ${ }^{25}$. In at least one case, a host SWEET gene is targeted by multiple TAL effectors from different strains of the pathogen: in addition to AvrXa7, OsSWEET14 is targeted by PthXo3, TalC and Tal5, each from a different Xoo strain ${ }^{23,28,50}$. The central role of SWEETs in susceptibility is further supported by our data, as we have identified GhSWEET10 as a clade III SWEET gene in cotton targeted by Avrb6. In addition, we observed that several different $\mathrm{Xcm}$ field isolates strongly induce two additional clade III GhSWEETs, GhSWEET14a and GhSWEET14b, but not GhSWEET10, during infections. Since $\mathrm{XcmH1005}$ is a strain derived from an older race, and since these field isolates were collected within the past year, this strongly suggests that new TAL effectors have evolved in these field isolates to target new SWEETs and break resistance. The striking differences of the tal gene content and the localization of the $p t h N 2$ gene on chromosome or plasmid in $\mathrm{XcmH1005}$ and $\mathrm{XcmN1003}$ are consistent with the notion that TAL effectors in $\mathrm{Xcm}$ may evolve rapidly. However, it is also possible that TAL effectors from these recent strains can activate another target, either another SWEET gene or a gene in different family, to cause susceptibility. It was revealed that TalC from Xoo can still cause disease on rice, despite having its corresponding EBE from OsSWEET14 artificially mutated, suggesting that preventing TalC-mediated SWEET14 activation alone is not sufficient to prevent susceptibility ${ }^{51}$. Additionally, even though GhSWEET10, GhSWEET14a and GhSWEET14b reside in clade III, it is still unclear if they function similarly. Additional biochemical analyses are needed to further understand the function of these genes in BBC. Characterizing the spectrum of SWEET-targeting TAL effectors and the relative contributions of their targets to susceptibility, both in the older strains and the recent isolates, is an important future objective.

The CRR of TAL effectors determines their DNA-binding specificity for targeted gene activation in disease or resistance. Inventory of the repeat sequences is essential to predict important TAL effector targets. However, the repeats are generally impossible to assemble from Illumina-sequencing reads and challenge remains even with Sanger sequencing. PacBio sequencing has proven to be an effective approach however ${ }^{52-54}$. We used PacBio sequencing to reveal the whole repertoire of TAL effectors in $\mathrm{XcmH} 1005$ and $\mathrm{XcmN1003}$. We identified 12 TAL effectors encoded by H1005 (six in the chromosome and six in the plasmid) and 9 encoded by N1003 (five in the chromosome and four in the plasmid). In addition to GhSWEET genes, another shared class of targets across TAL effectors of different Xanthomonas species is transcription factor genes ${ }^{19,31,55}$. In at least one case, the CsLOB1 transcription factor of citrus, and other members of CsLOB1 family also function as $S$ genes $^{31,56}$. It will be interesting to determine whether targets of other TAL effectors in $\mathrm{H} 1005$ and N1003 similarly include transcription factor genes, or yet novel types of targets.

In addition to revealing a host factor essential for disease symptom development, our study yields insight into the nature of BBC resistance mediated by $b 6$. Initially characterized as recessive ${ }^{57}$, genetic linkage mapping analysis suggests that the genetic basis of b6-resistance is rather complex and exhibits a quantitative inheritance. In rice, a mechanism of recessive resistance is disruption of TAL effector-dependent activation of an OsSWEET $S$ gene due to polymorphism at the $\mathrm{EBE}^{58}$. Our data revealed that the induction of GhSWEET10 by Avrb6 in Acb6 is compromised likely not due to the sequence polymorphism at the EBE, suggesting a novel mechanism underlying b6-mediated resistance. To our knowledge, this is the first report of polymorphisms outside the EBE itself that impair activation by a TAL effector. The $b 6$-mediated resistance is accompanied by the HR at the site of infection, and this is triggered specifically in the presence of Avrb6 (refs 10,38). However, a dTALE-activating GhSWEET10 only results in water-soaking, but not HR in Acb6, suggesting that GhSWEET10 does not mediate Avb6-induced HR. This is likely that HR is due to the activation of another target by Avrb6 in Acb6, but not in Ac44E. Thus, it seems possible that $b 6$ comprises at least two genetic determinants, one manifesting in reduced susceptibility (lower expression of the GhSWEET10 S gene) and the other mediating the defence-associated HR. Perhaps both need to be present to support the full level of resistance.

Nevertheless, our findings suggest a strategy to improve $\mathrm{BBC}$ resistance in cotton through genome editing of TAL effector EBEs in SWEET genes. Genome editing has been successfully used in rice to mutate the OsSWEET13 $S$ gene and generate plants resistant to Xoo strains that depend on TAL effector PthXo2 as a major virulence factor ${ }^{27}$. An advantage of this strategy is that relatively minor sequence changes could be made that will destroy the EBE while being unlikely to impinge on native promoter activity and without affecting the coding region, keeping endogenous function of the SWEET gene intact ${ }^{51,59}$. The successful knockout of all homeologs of the Mlo gene in hexaploid wheat in a single genome editing experiment indicates that disrupting EBEs in SWEET genes in cotton is likely feasible ${ }^{60}$. As new TAL effectors important for virulence of this pathogen are characterized in the future, this system could be used to edit the EBEs in the corresponding targets, whether GhSWEET genes or other BBC $S$ genes yet to be discovered, and to generate plants with broad-spectrum BBC resistance via pyramiding. Not least, duplicating the Acb6 genotype of GhSWEET10 in the Ac44E background and in other susceptible genotypes would be a powerful approach to dissect the genetic determinants of $b 6$-mediated resistance, potentially by uncoupling loss of susceptibility from the HR. Such characterizations would also likely lead to new strategies for improved control of BBC.

\section{Methods}

Plants and bacterial strains and growth conditions. The Gossypium hirsutum lines Acala44E (Ac44E), Acalab6 (Acb6) and FM706V, and G. arboreum line SA-1415 were grown in 3.5-inch square pots containing Metro Mix 900 soils (Sun Gro Horticulture, Agawam, MA) and tobacco Nicotiana benthamiana were grown in 3.5-inch square pots containing Metro Mix 366 soils in a growth chamber at $23{ }^{\circ} \mathrm{C}, 30 \%$ humidity and $100 \mu \mathrm{E} \mathrm{m} \mathrm{m}^{-2} \mathrm{~s}^{-1}$ light with a 12 -h light/12-h dark photoperiod. Two-week-old cotton plants were used for Agrobacterium-mediated VIGS assay, Agrobacterium-mediated transient protein expression assay or protoplast isolation. Plants after inoculation with Xanthomonas citri subsp. malvacearum $(X \mathrm{~cm})$ were transferred into a growth chamber at $28^{\circ} \mathrm{C}, 50 \%$ 
humidity and $100 \mu \mathrm{E} \mathrm{m}^{-2} \mathrm{~s}^{-1}$ light with a 12-h light/12-h dark photoperiod. Bacterial strains used in this study are listed in Supplementary Table 3. E. coli and A. tumefaciens strains were grown in Luria-Bertani (LB) medium with appropriate antibiotics at $37^{\circ} \mathrm{C}$ or $28^{\circ} \mathrm{C}$, respectively. $X \mathrm{~cm}$ strains were grown in nutrient broth medium with appropriate antibiotics at $28^{\circ} \mathrm{C}$. The $\mathrm{Xcm}$ field strains were isolated from infected cotton fields in Texas through Koch's postulates with modifications. Water-soaked lesions were excised from infected cotton leaves using a sterile scalpel. The cut lesions were soaked and macerated in $100 \mu \mathrm{l}$ of sterile water. The bacterial suspension was plated on nutrient agar from serial dilutions. Colonies were re-streaked and inoculated into cotton to confirm pathogenicity.

Xcm inoculations. The $\mathrm{Xcm}$ strains were inoculated into $4 \mathrm{ml}$ of nutrient broth medium with appropriate antibiotics for overnight. For syringe infiltration, bacterial suspensions adjusted to $\mathrm{OD}_{600}=0.1$ were inoculated into expanded cotyledons of 2-week-old plants or true leaves using a needleless syringe. Small holes were created with a $25 \mathrm{G}$ needle on the underside of the cotyledons to facilitate infiltration. For vacuum infiltration, a final bacterial suspension was made by adjusting the bacterial cultures to $\mathrm{OD}_{600}=0.01$ mixed with $0.04 \%$ of Silwet L-77 surfactant solution. Plants that were silenced via VIGS 3 weeks earlier were submerged into the bacterial suspension inside a desiccator connected to a vacuum pump. The plants were vacuum infiltrated for $5 \mathrm{~min}$ at $76 \mathrm{mmHg}$.

DNA isolation for $\mathbf{X c m}$ whole-genome sequencing. The $\mathrm{XcmH1005}$ and $X c m N 1003$ DNA used for PacBio sequencing was prepared using a protocol for total genomic DNA isolation with modification $\mathrm{s}^{54}$. Bacteria were cultured overnight in $30 \mathrm{ml}$ glucose yeast extract media ( $2 \%$ glucose, $1 \%$ yeast extract) in a $250 \mathrm{ml}$ flask at $28^{\circ} \mathrm{C}$ on a rotary shaker at $250 \mathrm{rpm}$, collected by centrifugation at $2,580 \mathrm{~g}$ for $10 \mathrm{~min}$ at $4^{\circ} \mathrm{C}$, then gently resuspended and washed in $20 \mathrm{ml} \mathrm{NE}$ buffer $(0.15 \mathrm{M} \mathrm{NaCl}, 50 \mathrm{mM}$ EDTA) twice to remove the extracellular polysaccharide. Cells were then gently resuspended in $2.5 \mathrm{ml}$ solution containing $50 \mathrm{mM}$ Tris, $\mathrm{pH} 8.0$ and $50 \mathrm{mM}$ EDTA, and then $0.5 \mathrm{ml}$ solution containing $25 \mathrm{mM}$ Tris, $\mathrm{pH} 8.0$, $10 \mu \mathrm{l}$ ReadyLyse (Epicentre) and $50 \mu \mathrm{l}$ RNase $\left(10 \mathrm{mg} \mathrm{ml}^{-1}\right)$. Suspensions were mixed gently by inversion and then incubated on ice for $45 \mathrm{~min}$. Following incubation on ice, $1.0 \mathrm{ml}$ STEP buffer $(0.5 \%$ SDS, $50 \mathrm{mM}$ Tris, pH 7.5, $40 \mathrm{mM}$ EDTA, protease $\mathrm{K}$ at $2 \mathrm{mg} \mathrm{ml}^{-1}$ ) was added, and the lysate was mixed well by inversion and incubated at $37^{\circ} \mathrm{C}$ for $1 \mathrm{~h}$, mixing every $10-15 \mathrm{~min}$. Next, $1.8 \mathrm{ml}$ of $7.5 \mathrm{M}$ ammonium acetate was added and the lysates were mixed gently before being subjected to two extractions with phenol/chloroform $(10 \mathrm{ml})$ and one extraction

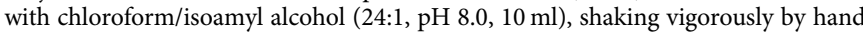
to mix and separating the aqueous and organic phases by centrifugation each time at $10,300 \mathrm{~g}$ for $10 \mathrm{~min}$ at $4{ }^{\circ} \mathrm{C}$. Following this, the aqueous phase was transferred to a $14 \mathrm{ml}$ tube and DNA was precipitated by addition of two volumes of cold $95 \%$ ethanol and gentle, repeated inversion. Once solidified, the DNA was transferred to a $2 \mathrm{ml} \mathrm{micro-centrifuge} \mathrm{tube} \mathrm{using} \mathrm{a} \mathrm{Pasteur} \mathrm{pipette} \mathrm{with} \mathrm{the} \mathrm{tip} \mathrm{previously} \mathrm{sealed}$ and bent into a hook over a flame. Remaining liquid was then removed by centrifugation at $825 \mathrm{~g}$ for $5 \mathrm{~min}$ and the pellet was washed once with $70 \%$ ethanol. The remaining liquid was removed as before and the tube left open to dry in a laminar flow hood until the edges of the pellet became glossy in appearance (10-15 min). Finally, the pellet was dissolved in $100 \mathrm{ml}$ TE buffer $(10 \mathrm{mM}$ Tris/HCl, $1 \mathrm{mM}$ EDTA, pH 8.0) overnight at $4{ }^{\circ} \mathrm{C}$ and then adjusted to a concentration of $1 \mu \mathrm{g} \mathrm{ml}^{-1}$.

PacBio sequencing and genome assembly. DNA library preparation and sequencing was performed according to the manufacturer's instructions by the Genomics Core Facility of the Icahn School of Medicine at Mt. Sinai (New York, NY, USA $)^{54}$. Separately, DNA preparation and electrophoresis to check for small plasmids in either strain that would be lost during library preparation was using standard methods ${ }^{50}$. Sequencing was conducted to $>120 \times$ coverage, which due to improvements in sequencing technology required only two SMRT cells per strain. In all data sets, read-length distribution showed a fat tail, with $20 \%$ of coverage after adaptor removal contained in subreads greater than or equal to $15,000 \mathrm{bp}$. The whole genomes of H1005 and N1003 were assembled using HGAP3 (ref. 61) and verified by carrying out local assemblies of reads containing tal genes and then comparing those assemblies to the whole-genome assembly as described in Booher et al. ${ }^{54}$. The assemblies conclusively match published Southern blot results using a tal gene probe, as well as restriction enzyme mapping and sequencing or partial sequencing of cosmid clones and subclones ${ }^{10,38-40}$.

The initial assembly of $\mathrm{XcmH} 1005$ showed frameshifts in avrB104, avr103 and avrb6 resulting from an extra cytosine (C) in a stretch of six Cs commonly found in the $3^{\prime}$ region of tal genes. Since avrb6 is functional, the previously published sequence of avrb6 shows an intact reading frame ${ }^{38}$, and residual errors in homopolymer runs sometimes persist after consensus calling by Quiver and are a known weak point of the error-correction software (https://github.com/ PacificBiosciences/GenomicConsensus/blob/master/doc/FAQ.rst), we concluded that the frameshift in avrb6 was such an error, and we corrected it in the final assembly. Likewise, since clones containing avrB104 and avrB103 were functional, conferring avirulence on $A c B 5$ and $A c B I n 3$-containing $\operatorname{cotton}^{40}$, we concluded that the assembly contained the same error for each of these genes, and corrected them both. No other tal genes in the assembly harboured this or another frameshift within a homopolymer stretch. Note that the frameshift in $p t h N$, retained in the final assembly, is due to a deletion of a single thymine, not within a homopolymer, and $p t h N^{\prime}$ was shown to be non-functional ${ }^{10}$

Construction of avrb6 in the expression vectors. Avrb6 was amplified from $\mathrm{Xcm} \mathrm{H} 1005$ plasmid DNA with primers containing restriction enzymes SpeI at the amino $(\mathrm{N})$ terminus and SmaI at the carboxy $(\mathrm{C})$ terminus (Supplementary Table 4) and ligated into a plant protoplast expression vector $p H B T$ with a $C a M V$ $35 S$ promoter at the $\mathrm{N}$ terminus and a HA-epitope tag at the $\mathrm{C}$ terminus. To construct the $p C B 302$ binary vector containing avrb6 for Agrobacterium-mediated transient expression assay in $N$. benthamiana, avrb6 was released from the $p H B T$ vector through digestion with SpeI and SmaI and ligated into the $p$ CB302 binary vector. All the clones were confirmed by sequencing.

Transient expression assays. Cotton protoplasts were isolated from 2-week-old cotyledons. Detached cotyledons were cut with a razor blade and digested in an enzyme solution ( $1.5 \%$ cellulose R10, $0.4 \%$ macerozyme R10, 0.4 M mannitol, $20 \mathrm{mM} \mathrm{KCl}, 20 \mathrm{mM}$ MES pH 5.7) supplemented with $2 \%$ sucrose for $0.5 \mathrm{~h}$ under vacuum. Subsequently, the enzyme solution was incubated without vacuum at room temperature for $12 \mathrm{~h}$. Protoplasts were released by filtering through a $30 \mu \mathrm{m}$-nylon mesh, washed with W5 solution $(154 \mathrm{mM} \mathrm{NaCl}, 125 \mathrm{mM} \mathrm{CaCl}$, $5 \mathrm{mM} \mathrm{KCl}, 2 \mathrm{mM}$ MES pH 5.7) and diluted in MMG solution (0.4 M mannitol, $15 \mathrm{mM} \mathrm{MgCl}_{2}, 4 \mathrm{mM}$ MES pH 5.7) to a density of $2 \times 10^{5} \mathrm{cells} \mathrm{ml}^{-1}$ (ref. 62). A measure of $100 \mu \mathrm{l}$ of protoplasts for each sample were collected for western blotting after expressing avrb6-HA for $12 \mathrm{~h}$. Avrb6-HA protein was detected by immunoblotting using the $\alpha$-HA antibody. Agrobacterium tumefaciens strain GV3101 containing pCB302-avrb6-HA vector or an empty vector was cultured overnight in LB medium containing appropriate antibiotics at $28^{\circ} \mathrm{C}$. Bacteria were collected by centrifugation and resuspended with infiltration buffer $(10 \mathrm{mM}$ MES, $\mathrm{pH} 5.7,10 \mathrm{mM} \mathrm{MgCl}_{2}, 200 \mu \mathrm{M}$ acetosyringone) at $\mathrm{OD}_{600}=0.6$. Leaves of 4-week-old soil-grown N. benthamiana or cotyledons of 2-week-old Ac44E cotton were hand infiltrated using a needleless syringe with Agrobacterium cultures. Leaf samples were collected $48 \mathrm{~h}$ after infiltration for protein sample and immunoblot analysis. Full blots are provided in Supplementary Fig. 10.

Cotton RNA isolation for RT-PCR and qRT-PCR analysis. Cotton total RNA was extracted from protoplasts $\left(500 \mu\right.$ l protoplasts with a cell density of $2 \times 10^{5}$ per ml for each sample) using TRIzol reagent (Invitrogen, USA) or leaves (one cotyledon or one true leaf for each sample) using a Spectrum plant total RNA kit (Sigma-Aldrich, USA) according to the manufacturer's protocol. RNA was then treated with DNase (Invitrogen, USA) to remove genomic DNA. Purified RNA $(1 \mu \mathrm{g})$ was subsequently reverse transcribed using a first-strand cDNA synthesis kit (Promega, USA) with oligo(dT) as a primer. PCR amplification was performed for 30 cycles of $15 \mathrm{~s}$ at $98^{\circ} \mathrm{C}, 30 \mathrm{~s}$ at $55^{\circ} \mathrm{C}$ and $45 \mathrm{~s}$ at $72^{\circ} \mathrm{C}$ with primers listed in Supplementary Table 4. GhACTIN was used as an internal control for RT-PCR. qRT-PCR analysis was performed using iTaq SYBR green Supermix (Bio-Rad, USA) with a Bio-Rad CFX384 Real-Time PCR System. Expression of each gene was normalized to the expression of GhUBQ1.

RNA sequencing and data analysis. A measure of $500 \mu \mathrm{l}$ of cotton protoplasts $\left(2 \times 10^{5}\right.$ per ml $)$ were transfected with 35 S::avrb6-HA or an empty vector control Protoplasts were collected $12 \mathrm{~h}$ after transfection and RNA was extracted by the TRIzol reagent. Three independent biological repeats were performed for the RNA-Seq analysis. For each repeat, equal amount of RNA from two biological replicates was pooled for RNA-Seq library construction. RNA-Seq libraries were prepared using Illumina TruSeqTM RNA Sample Preparation Kit following the manufacturer's instruction, and sequenced on an Illumina HiSeq 2000 platform with 125-nucleotide pair-end reads at Texas AgriLife Genomics and Bioinformatics Service (College Station, TX). RNA-Seq read processing, alignment to the G. raimondii genome and differential gene expression analysis were performed as described in Li et al. ${ }^{63}$. Genes with expression fold change $\geq 2$ and $P$ value $<0.05$ were considered as significantly different between samples with and without Avrb6.

EBE prediction. All TAL effector EBE predictions were conducted using the TALE-NT 2.0 Target Finder tool ${ }^{64}$ to search the promoter regions defined in the G. raimondii genome as the 1,000 bp upstream of the transcriptional start site plus the $5^{\prime}$ UTR, if annotated. We identified promoter sequences for G. raimondii using genome assembly v2.0 and annotation v2.1 (ref. 3). Binding sites passing the standard target finder score ratio cutoff of 3.0 were ranked based on both target finder output and genomic context using the machine-learning classifier presented by Cernadas et al. ${ }^{55}$ and updated by Wilkins et al. ${ }^{65}$.

Construction of VIGS vector and Agrobacterium-mediated VIGS. GhSWEET10 was amplified by PCR from cDNA of G. hirsutum with primers described in Supplementary Table 4, and inserted into the pYL156 (pTRV-RNA2) vector with restriction enzymes EcoRI and KpnI, resulting in pYL156-GhSWEET10. The Agrobacterium tumefaciens strain GV3101 carrying the plasmids pTRV-RNA1 and pYL156 (pTRV-RNA2) or pYL156-GhSWEET10 was collected and resuspended 
with infiltration buffer (10 mM MES, pH 5.7, $10 \mathrm{mM} \mathrm{MgCl}_{2}, 200 \mu \mathrm{M}$ acetosyringone) at $\mathrm{OD}_{600}=0.75$. The culture suspension of pTRV-RNA1 was mixed with pYL156 or pYL156-GhSWEET10 at a 1:1 ratio and hand infiltrated using needleless syringes into two fully expanded cotyledons of 2-week-old plants ${ }^{66}$.

GhSWEET10D transporter analysis in HEK293T cells. HEK293T cells were co-transfected with FRET sucrose sensor FLIPsuc $90 \mu \delta 3 \mathrm{~A}$ and GhSWEET10D in six-well plates and perfusion experiments were performed ${ }^{48}$. After $3.5 \mathrm{~min}$ buffer perfusion, cells were perfused with $25 \mathrm{mM}$ sucrose for 4 min followed by $2.5 \mathrm{~min}$ buffer perfusion. Images were captured at the following settings: exposure time $800 \mathrm{~ms}$, gain 3, binning 2, time interval $10 \mathrm{~s}$. Fluorescence intensity of selected individual cells was recorded. The quantitative ratio of eCFP and Aphrodite emission at excitation of eCFP was calculated after the background was subtracted. All of ratios were normalized to the initial ratio.

Yeast mutant EBY4000 complementation growth assay. The yeast strain EBY4000 was transformed with the different plasmids following the standard protocol. Spotting assay with 10 -fold serial dilutions was performed. Yeast cells were diluted to $\mathrm{OD}_{600}=0.22$ in water after cells grew to $\mathrm{OD}_{600}=0.6$, followed by a twofold dilution. Then, 10 -fold serial dilutions were plated on synthetic medium containing either $2 \%$ maltose (as a control), $2 \%$ fructose or $2 \%$ glucose. Growth was documented by Gel-doc system after $2-4$ days at $30{ }^{\circ} \mathrm{C}$.

Transactivation assays. The reporter constructs, $p$ GhSWEET10D::LUC, pGh067700::LUC, pGhKBS1::LUC, pGhMDR1::LUC, pGhHLH1::LUC and pGaSWEET10::LUC, were constructed by amplifying the promoters of candidate genes ( $\sim 800$ bp upstream of translational start site) from genomic DNA of Ac44E, Acb6 or G. arboreum and were fused to a luciferase gene at the $\mathrm{C}$ terminus. Protoplasts were transfected with the effector and reporter construct and expressed at room temperature for $12 \mathrm{~h}$. Protoplasts were then collected and lysed and the luciferase activity was detected by Glomax Multi-Detection System (Promega, USA) with the luciferase assay substrate (Promega, USA). UBQ10-GUS was included in all samples as the internal transfection control and the GUS activity was analysed with a Multilabel Plate Reader (Perkin-Elmer, USA). The $p$ GhSWEET10D-mEBE::LUC construct was generated by the site-directed mutagenesis PCR using plasmid DNA of $p$ GhSWEET10D::LUC as the template and primers are listed in Supplementary Table 4.

Construction of dTALEs. The dTALEs were constructed using golden gate cloning using a complete plasmid kit available through AddGene ${ }^{67}$. Four types of repeats encoding the RVDs NI, NN, NG and HD that correspond to the respective nucleotide $A, G, T$ and $C$ were used to assemble the repeat domains of the artificial dTALEs. The dTALEs were transformed into $\mathrm{Xcm}$ stains via bacterial tri-parental mating by using a helper plasmid, pRK2073 (ref. 38). In addition, the modifier plasmid pUFR054 was used to increase the efficiency of transferring the plasmids from E. coli DH5 $\alpha$ to $\mathrm{Xcm}$ strains. The bacterial mating was carried out by mixing the donor, recipient, modifier and helper strains in a 1:1:1:1 ratio. The mixed culture was plated onto nutrient agar medium with appropriate antibiotics. Colonies were confirmed via Sanger sequencing of extracted plasmid DNA.

Measurement of sucrose concentration. Leaves from 4-week-old $N$. benthamiana plants were infiltrated with Agrobacterium carrying 35S::GhSWEET1OD-HA or an empty vector control at $\mathrm{OD}_{600}=1.0$. Leaves were detached $3 \mathrm{dpi}$ and fully vacuum infiltrated with sterile Millipore water. The leaves were blot dried, cut into small pieces with a razor blade and placed in a nylon mesh strainer at the top of a $50 \mathrm{ml}$ centrifuge tube. The apoplastic solution was collected through centrifugation at $3,220 \mathrm{~g}$ for $20 \mathrm{~min}$ at $4^{\circ} \mathrm{C}$. The concentration of sucrose was determined by using a sucrose assay kit (Sigma-Aldrich).

Data availability. The $\mathrm{Xcm} H 1005$ genome assembly has been deposited in GenBank under accessions CP013004 (chromosome) and CP013005 (pXcmH) and the XcmN1003 assembly under accessions CP013006 (chromosome) and CP013007 (pXcmN). Raw data as bas.h5 and bax.h5 files and associated metadata are available in the NCBI sequence read archive (SRA) under SRP065335 for XcmH and SRP065336 for $X \mathrm{~cm}$ N. The RNA-Seq data were deposited in the NCBI SRA under accession number SRP100553. The authors declare that all other data supporting the findings of this study are included within the manuscript and its supplementary files or are available from the corresponding authors on request.

\section{References}

1. Paterson, A. H. et al. Repeated polyploidization of Gossypium genomes and the evolution of spinnable cotton fibres. Nature 492, 423-427 (2012).

2. Wendel, J. F. New world tetraploid cottons contain old world cytoplasm. Proc. Natl Acad. Sci. USA 86, 4132-4136 (1989).

3. Wang, K. et al. The draft genome of a diploid cotton Gossypium raimondii. Nat. Genet. 44, 1098-1103 (2012).

4. Li, F. et al. Genome sequence of the cultivated cotton Gossypium arboreum. Nat. Genet. 46, 567-572 (2014).
5. Li, F. et al. Genome sequence of cultivated upland cotton (Gossypium hirsutum TM-1) provides insights into genome evolution. Nat. Biotechnol. 33, 524-530 (2015).

6. Zhang, T. et al. Sequencing of allotetraploid cotton (Gossypium hirsutum L. acc. TM-1) provides a resource for fiber improvement. Nat. Biotechnol. 33, 531-537 (2015).

7. Liu, X. et al. Gossypium barbadense genome sequence provides insight into the evolution of extra-long staple fiber and specialized metabolites. Sci. Rep. 5, 14139 (2015)

8. Yuan, D. et al. The genome sequence of Sea-Island cotton (Gossypium barbadense) provides insights into the allopolyploidization and development of superior spinnable fibres. Sci. Rep. 5, 17662 (2015).

9. Delannoy, E. et al. Resistance of cotton towards Xanthomonas campestris pv. malvacearum. Annu. Rev. Phytopathol. 43, 63-82 (2005).

10. Chakrabarty, P. K., Duan, Y. P. \& Gabriel, D. W. Cloning and characterization of a member of the Xanthomonas avr/pth gene family that evades all commercially utilized cotton R genes in the United States. Phytopathology 87, 1160-1167 (1997).

11. Bogdanove, A. J., Schornack, S. \& Lahaye, T. TAL effectors: finding plant genes for disease and defense. Curr. Opin. Plant Biol. 13, 394-401 (2010).

12. Boch, J. \& Bonas, U. Xanthomonas AvrBs3 family-type III effectors: discovery and function. Annu. Rev. Phytopathol. 48, 419-436 (2010).

13. Mak, A. N. et al. The crystal structure of TAL effector PthXol bound to its DNA target. Science 335, 716-719 (2012).

14. Deng, D. et al. Structural basis for sequence-specific recognition of DNA by TAL effectors. Science 335, 720-723 (2012).

15. Schreiber, T. \& Bonas, U. Repeat 1 of TAL effectors affects target specificity for the base at position zero. Nucleic Acids Res. 42, 7160-7169 (2014).

16. Doyle, E. L. et al. TAL effector specificity for base 0 of the DNA target is altered in a complex, effector- and assay-dependent manner by substitutions for the tryptophan in cryptic repeat -1. PLoS ONE 8, e82120 (2013).

17. Marois, E., Van den Ackerveken, G. \& Bonas, U. The Xanthomonas type III effector protein AvrBs3 modulates plant gene expression and induces cell hypertrophy in the susceptible host. Mol. Plant Microbe Interact. 15, 637-646 (2002).

18. Römer, P. et al. Plant pathogen recognition mediated by promoter activation of the pepper Bs3 resistance gene. Science 318, 645-648 (2007).

19. Kay, S. et al. A bacterial effector acts as a plant transcription factor and induces a cell size regulator. Science 318, 648-651 (2007).

20. Yang, B., Sugio, A. \& White, F. F. Os8N3 is a host disease-susceptibility gene for bacterial blight of rice. Proc. Natl Acad. Sci. USA 103, 10503-10508 (2006).

21. Chu, Z. H. et al. Promoter mutations of an essential gene for pollen development result in disease resistance in rice. Genes Dev. 20, 1250-1255 (2006).

22. Yuan, M. et al. Pathogen-induced expressional loss of function is the key factor in race-specific bacterial resistance conferred by a recessive $\mathrm{R}$ gene xal3 in rice. Plant Cell Physiol. 50, 947-955 (2009).

23. Antony, G. et al. Rice xa13 recessive resistance to bacterial blight is defeated by induction of the disease susceptibility gene Os-11N3. Plant Cell 22, 3864-3876 (2010).

24. Kay, S. et al. Detailed analysis of the DNA recognition motifs of the Xanthomonas type III effectors AvrBs3 and AvrBs3Deltarep16. Plant J. 59, 859-871 (2009).

25. Cohn, M. et al. Xanthomonas axonopodis virulence is promoted by a transcription activator-like effector-mediated induction of a SWEET sugar transporter in cassava. Mol. Plant Microbe Interact. 27, 1186-1198 (2014).

26. Huang, S. et al. The broadly effective recessive resistance gene xa5 of rice is a virulence effector-dependent quantitative trait for bacterial blight. Plant J. 86, 186-194 (2016)

27. Zhou, J. et al. Gene targeting by the TAL effector PthXo2 reveals cryptic resistance gene for bacterial blight of rice. Plant J. 82, 632-643 (2015).

28. Streubel, J. et al. Five phylogenetically close rice SWEET genes confer TAL effector-mediated susceptibility to Xanthomonas oryzae pv. oryzae. New Phytol. 200, 808-819 (2013).

29. Romer, P. et al. Promoter elements of rice susceptibility genes are bound and activated by specific TAL effectors from the bacterial blight pathogen, Xanthomonas oryzae pv. oryzae. New Phytol. 187, 1048-1057 (2010).

30. Chen, L. Q. SWEET sugar transporters for phloem transport and pathogen nutrition. New Phytol. 201, 1150-1155 (2014).

31. Hu, Y. et al. Lateral organ boundaries 1 is a disease susceptibility gene for citrus bacterial canker disease. Proc. Natl Acad. Sci. USA 111, E521-E529 (2014).

32. Gu, K. et al. R gene expression induced by a type-III effector triggers disease resistance in rice. Nature 435, 1122-1125 (2005).

33. Wang, C. et al. XA23 is an executor R protein and confers broad-spectrum disease resistance in rice. Mol. Plant 8, 290-302 (2015).

34. Tian, D. et al. The rice TAL effector-dependent resistance protein XA10 triggers cell death and calcium depletion in the endoplasmic reticulum. Plant Cell 26, 497-515 (2014). 
35. Cernadas, R. A. et al. Code-assisted discovery of TAL effector targets in bacterial leaf streak of rice reveals contrast with bacterial blight and a novel susceptibility gene. PLoS Pathog. 10, e1003972 (2014).

36. Strauss, T. et al. RNA-seq pinpoints a Xanthomonas TAL-effector activated resistance gene in a large-crop genome. Proc. Natl Acad. Sci. USA 109, 19480-19485 (2012).

37. Yang, Y. N., Defeyter, R. \& Gabriel, D. W. Host-specific symptoms and increased release of Xanthomonas citri and Xanthomonas campestris pv. malvacearum from leaves are determined by the 102-bp tandem repeats of PthA and Avrb6, respectively. Mol. Plant Microbe Interact. 7, 345-355 (1994).

38. DeFeyter, R., Yang, Y. O. \& Gabriel, D. W. Gene-for-genes interactions between cotton R genes and Xanthomonas campestris pv. malvacearum Avr genes. Mol. Plant Microbe Interact. 6, 225-237 (1993).

39. DeFeyter, R. \& Gabriel, D. W. At least six avirulence genes are clustered on a 90-kilobase plasmid in Xanthomonas campestris pv. malvacearum. Mol. Plant Microbe Interact. 4, 423-432 (1991).

40. Yang, Y., Yuan, Q. \& Gabriel, D. W. Watersoaking function(s) of XcmH1005 are redundantly encoded by members of the Xanthomonas avr/pth gene family. Am. Phytopathol. Soc. 9, 105-113 (1996).

41. Salzberg, S. L. et al. Genome sequence and rapid evolution of the rice pathogen Xanthomonas oryzae pv. oryzae PXO99A. BMC Genomics 9, 204 (2008).

42. Essenberg, M. et al. Four near-isogenic lines of cotton with different genes for bacterial blight resistance. Phytopathology 92, 1323-1328 (2002).

43. Meckler, J. F. et al. Quantitative analysis of TALE-DNA interactions suggests polarity effects. Nucleic Acids Res. 41, 4118-4128 (2013).

44. Knight, R. L. \& Hutchinson, J. B. The evolution of blackarm resistance in cotton. J. Genet. 50, 36-58 (1950).

45. Hou, B. H. et al. Optical sensors for monitoring dynamic changes of intracellular metabolite levels in mammalian cells. Nat. Protoc. 6, 1818-1833 (2011).

46. Eom, J. S. et al. SWEETs, transporters for intracellular and intercellular sugar translocation. Curr. Opin. Plant Biol. 25, 53-62 (2015).

47. Talbot, N. J. Cell biology: raiding the sweet shop. Nature 468, 510-511 (2010).

48. Chen, L. Q. et al. Sucrose efflux mediated by SWEET proteins as a key step for phloem transport. Science 335, 207-211 (2012).

49. Kanno, Y. et al. AtSWEET13 and AtSWEET14 regulate gibberellin-mediated physiological processes. Nat. Commun. 7, 13245 (2016).

50. Yu, Y. et al. Colonization of rice leaf blades by an African strain of Xanthomonas oryzae pv. oryzae depends on a new TAL effector that induces the rice nodulin-3 Os11N3 gene. Mol. Plant Microbe Interact. 24, 1102-1113 (2011).

51. Blanvillain-Baufume, S. et al. Targeted promoter editing for rice resistance to Xanthomonas oryzae pv. oryzae reveals differential activities for SWEET14inducing TAL effectors. Plant Biotechnol. J. 15, 306-317 (2017).

52. Huguet-Tapia, J. C. et al. Complete genome sequence of the African strain AXO1947 of Xanthomonas oryzae pv. oryzae. Genome Announc. 4, e01730-15 (2016).

53. Grau, J. et al. AnnoTALE: bioinformatics tools for identification, annotation, and nomenclature of TALEs from Xanthomonas genomic sequences. Sci. Rep. 6, 21077 (2016)

54. Booher, N. J. et al. Single molecule real-time sequencing of Xanthomonas oryzae genomes reveals a dynamic structure and complex TAL (transcription activator-like) effector gene relationships. Microb. Genom. 1, doi:10.1099/ mgen.1090.000032 (2015).

55. Sugio, A. et al. Two type III effector genes of Xanthomonas oryzae pv. oryzae control the induction of the host genes OsTFIIAgammal and OsTFX1 during bacterial blight of rice. Proc. Natl Acad. Sci. USA 104, 10720-10725 (2007).

56. Zhang, J. et al. Homologs of CsLOB1 in citrus function as disease susceptibility genes in citrus canker. Mol. Plant Pathol. doi:10.1111/mpp.12441 (2016).

57. Wright, R. J. et al. D-subgenome bias of $\mathrm{Xcm}$ resistance genes in tetraploid Gossypium (cotton) suggests that polyploid formation has created novel avenues for evolution. Genetics 149, 1987-1996 (1998).

58. Hutin, M. et al. MorTAL Kombat: the story of defense against TAL effectors through loss-of-susceptibility. Front. Plant Sci. 6, 535 (2015).

59. Li, T. et al. High-efficiency TALEN-based gene editing produces diseaseresistant rice. Nat. Biotechnol. 30, 390-392 (2012).

60. Wang, Y. et al. Simultaneous editing of three homoeoalleles in hexaploid bread wheat confers heritable resistance to powdery mildew. Nat. Biotechnol. 32, 947-951 (2014).
61. Chin, C. S. et al. Nonhybrid, finished microbial genome assemblies from longread SMRT sequencing data. Nat. Methods 10, 563-569 (2013).

62. Gao, X. et al. Silencing GhNDR1 and GhMKK2 compromises cotton resistance to Verticillium wilt. Plant J. 66, 293-305 (2011).

63. Li, F. et al. Modulation of RNA polymerase II phosphorylation downstream of pathogen perception orchestrates plant immunity. Cell Host Microbe 16, 748-758 (2014).

64. Doyle, E. L. et al. TAL effector-nucleotide targeter (TALE-NT) 2.0: tools for TAL effector design and target prediction. Nucleic Acids Res. 40, W117-W122 (2012).

65. Wilkins, K. E. et al. TAL effectors and activation of predicted host targets distinguish Asian from African strains of the rice pathogen Xanthomonas oryzae pv. oryzicola while strict conservation suggests universal importance of five TAL effectors. Front. Plant Sci. 6, 536 (2015).

66. Gao, X. et al. Cotton GhBAK1 mediates Verticillium wilt resistance and cell death. J. Integr. Plant Biol. 55, 586-596 (2013).

67. Cermak, T. et al. Efficient design and assembly of custom TALEN and other TAL effector-based constructs for DNA targeting. Nucleic Acids Res. 39, e82 (2011).

68. Darling, A. E., Mau, B. \& Perna, N. T. ProgressiveMauve: multiple genome alignment with gene gain, loss and rearrangement. PLoS ONE 5, e11147 (2010).

69. Tamura, K. et al. MEGA6: molecular evolutionary genetics analysis version 6.0 Mol. Biol. Evol. 30, 2725-2729 (2013).

\section{Acknowledgements}

We thank Dr Dean Gabriel for various Xcm strains, Dr Margaret Essenberg and Jared Harris for the seeds, Stephanie Yamashita and Tri Tran for excellent technical assistance and Karla Vogel for constructing pKEB1. This work was supported by the USDA NIFA (2012-67013-19433) to P.H. and L.S., the Cotton Inc (15-174) to L.S., Texas AgriLife Research Cotton Improvement Program to L.S., P.H. and T.W., the US National Science Foundation (Plant Genome Research Program Award IOS 1238189) to A.J.B., the Texas A\&M University Graduate Diversity Fellowship and USDA NIFA AFRI Pre-doctoral Fellowship (Award No. 2017-67011-26060) to K.L.C., Graduate Research Fellowship Award (Grant No. DGE-1144153) to K.E.W.

\section{Author contributions}

K.L.C., F.M., K.E.W., P.H., A.B., L.S. conceived the study and designed the experiments; K.C., F.M., K.W., F.L., P.W., N.J.B., S.C.D.C., L.-Q.C., H.Z., X.G., Y.Z., and Z.F. performed the experiments and/or data analysis; and K.C., P.H., A.J.B. and L.S. drafted the manuscript. The manuscript and figures were reviewed and revised by all the authors

\section{Additional information}

Supplementary Information accompanies this paper at http://www.nature.com/ naturecommunications

Competing interests: The authors declare no competing financial interests.

Reprints and permission information is available online at http://npg.nature.com/ reprintsandpermissions/

How to cite this article: Cox, K. L. et al. TAL effector driven induction of a SWEET gene confers susceptibility to bacterial blight of cotton. Nat. Commun. 8, 15588 doi: $10.1038 /$ ncomms15588 (2017).

Publisher's note: Springer Nature remains neutral with regard to jurisdictional claims in published maps and institutional affiliations.

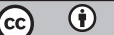

This work is licensed under a Creative Commons Attribution 4.0 International License. The images or other third party material in this article are included in the article's Creative Commons license, unless indicated otherwise in the credit line; if the material is not included under the Creative Commons license, users will need to obtain permission from the license holder to reproduce the material. To view a copy of this license, visit http://creativecommons.org/licenses/by/4.0/

(C) The Author(s) 2017 